\title{
Les sites ecclésiaux et monastiques de l'archipel du Kvarner (Croatie)
}

Campagne 2016

Morana Čaušević-Bully, Sébastien Bully, Jessy Crochat, Pascale Chevalier, Ivan Valent, Hervé Richard, Émilie Gauthier et Vincent Bichet

\section{(2) OpenEdition}

Journals

Édition électronique

URL : http://journals.openedition.org/cefr/1797

DOI : $10.4000 /$ cefr. 1797

ISSN : 2282-5703

Éditeur

École française de Rome

Référence électronique

Morana Čaušević-Bully, Sébastien Bully, Jessy Crochat, Pascale Chevalier, Ivan Valent, Hervé Richard, Émilie Gauthier et Vincent Bichet, « Les sites ecclésiaux et monastiques de l'archipel du Kvarner (Croatie) », Chronique des activités archéologiques de l'École française de Rome [En ligne], Balkans, mis en ligne le 24 août 2017, consulté le 23 mars 2020. URL : http://journals.openedition.org/cefr/1797 ; DOl : https://doi.org/10.4000/cefr.1797

Ce document a été généré automatiquement le 23 mars 2020.

(c) École française de Rome 


\section{Les sites ecclésiaux et monastiques de l'archipel du Kvarner (Croatie)}

Campagne 2016

Morana Čaušević-Bully, Sébastien Bully, Jessy Crochat, Pascale Chevalier, Ivan Valent, Hervé Richard, Émilie Gauthier et Vincent Bichet

\section{NOTE DE L'AUTEUR}

Avec la collaboration et les contributions de Thomas Chenal, Adrien Saggese, Lucija Dugorepec, Inès Pactat, Valentin Chevassu, Mathieu Thivet et Agnès Stock.

1 La campagne 2016 du programme de recherche sur les sites ecclésiaux et monastiques de l'archipel du Kvarner ${ }^{1}$ a essentiellement porté sur la fouille du petit complexe antique et médiéval septentrional du site de Mirine-Fulfinum - secteur dit de "l'église à trois absides » - (île de Krk), du 11 au 23 avril 2016, et sur la poursuite de la fouille de la grande église paléochrétienne du complexe de Martinšćica (île de Cres) du 27 juin au 14 juillet $^{2}$ (fig. 1). 
Fig. 1 - Carte de l'archipel du Kvarner et localisation des deux sites étudiés en 2016.



2 La reprise de la fouille du secteur de la petite église nord de Mirine-Fulfinum a permis de mieux circonscrire le complexe antique - probable villa suburbaine - dans lequel elle fut édifiée, tout en doutant désormais d'une fonction monastique pour privilégier l'hypothèse d'une église votive (des $\mathrm{IX}^{\mathrm{e}}-\mathrm{XI}^{\mathrm{e}} \mathrm{s}$.), sans lien direct avec la villa, vraisemblablement abandonnée dès le $\mathrm{VI}^{\mathrm{e}} \mathrm{s}$.

3 À Martinšćica, la seconde campagne de fouille sur la grande église nous a permis de documenter des installations liturgiques élaborées - en dépit d'un médiocre état de conservation - et de recenser un mobilier liturgique de qualité, nous interpellant sur le statut de l'édifice, avec, plus particulièrement, la découverte d'un monogramme sur un fragment de mensa en marbre. La fouille a également révélé deux sacristies sur le flanc nord de l'église. L'une d'elle conserve un magnifique pavement de mosaïques géométriques, scellé par un niveau d'occupation (foyer) induisant un très probable changement de fonction. Et c'est également une occupation domestique que l'on tend à attribuer à l'annexe greffée contre l'abside centrale découverte en 2015.

Dans le dessein d'une appréhension plus large des sites étudiés, nous avons également engagé cette année de premiers forages destinés aux analyses paléoenvironnementales des îles de Krk et Cres.

\section{Le « complexe ecclésial » de Mirine-Fulfinum (Omišalj, île de Krk)}

5 Après les travaux préparatoires réalisés au mois d'octobre $2015^{3}$, la première campagne de fouilles archéologiques a porté sur le secteur dit de «l'église à trois absides ». Ce volet du programme sur les sites ecclésiaux de l'archipel du Kvarner a été organisé dans 
le cadre d'un chantier école de l'Université de Franche-Comté et participe au projet de réalisation du parc archéologique de Mirine-Fulfinum initié par la commune d'Omišalji

Situé à environ $150 \mathrm{~m}$ au nord du complexe paléochrétien de Mirine (fig. 2), les recherches anciennes et des prospections géophysiques réalisées par nos soins en 2012 avaient permis de reconnaître un complexe antique - doté d'un petit balnéaire - et une petite église à trois absides de datation incertaine (entre le $\mathrm{IX}^{\mathrm{e}}$ et le $\mathrm{XI}^{\mathrm{e}} \mathrm{s}$.), construite au sein $\mathrm{du}$ complexe ${ }^{5}$. On rappellera que l'un des objectifs des recherches est de déterminer la nature de l'église et du complexe, leurs potentielles relations et les phases d'occupation. Pour ce faire, une attention soutenue est portée à la chronologie relative des maçonneries et à la stratigraphie.

7 En parallèle de la fouille nous avons engagé une campagne d'informatisation et de modalisation 3D de l'église et des mausolées paléochrétiens de Mirine ${ }^{6}$.

Fig. 2 - Mirine-Fulfinum, plan topographique général des vestiges (d'après M. Čaušević-Bully)

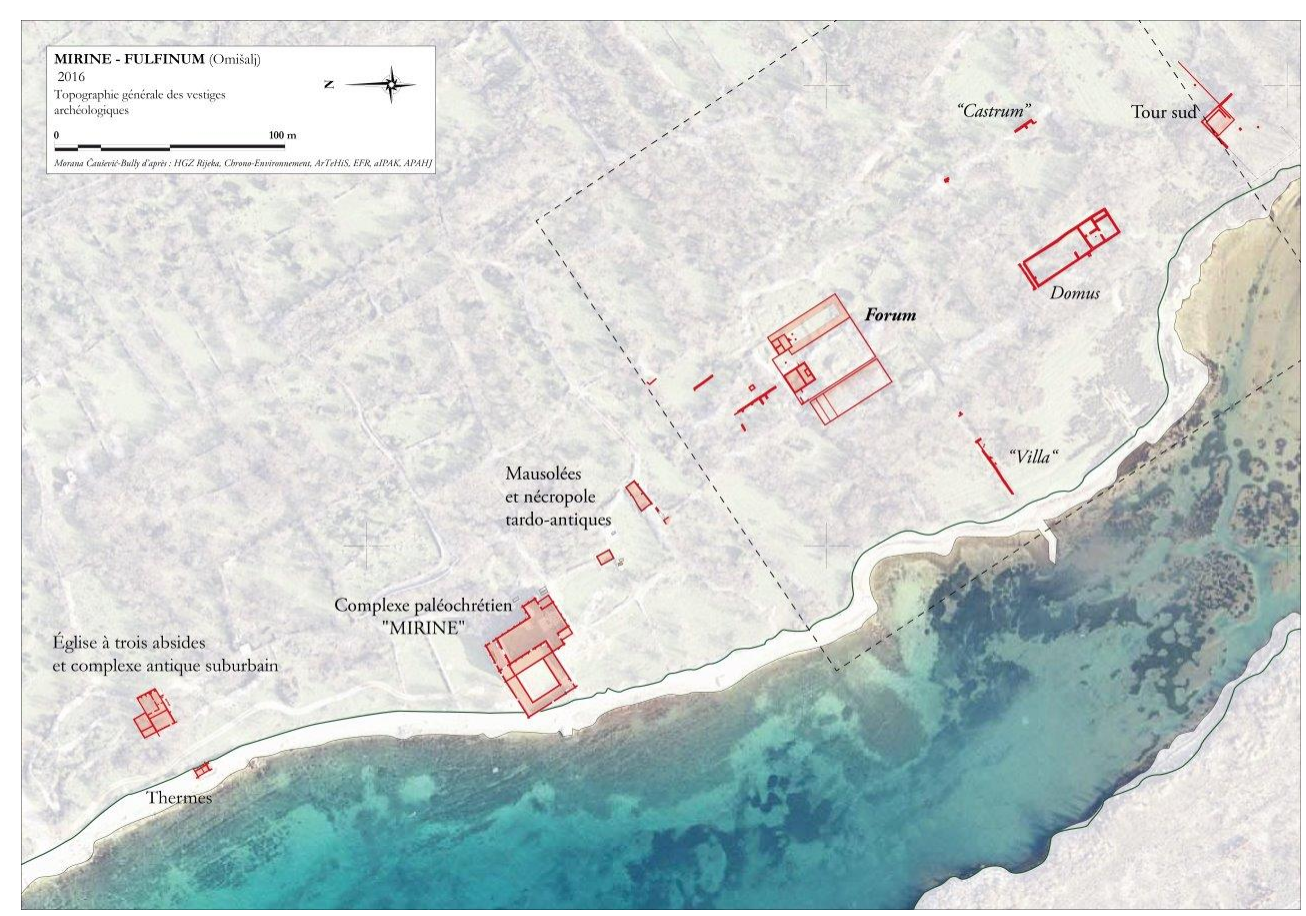

\section{Un complexe antique suburbain}

Le complexe antique suburbain est construit au bord de la mer et s'organise en terrasses, suivant selon la pente naturelle du terrain inclinée du nord-est au sud-ouest ; il s'organise autour d'une partie centrale composée des murs: nord-est 4.100, qui semble être le mur chevet du complexe, nord-ouest 4.101, sud-ouest 4.102, sud-est 4.127 et 4.103 (fig. 3). Tous les murs sont chaînés, dotés de talons de fondations réguliers, débordant de deux côtés d'une dizaine de $\mathrm{cm}$ de largeur en moyenne. Les élévations, construites en moellons sommairement équarris et disposés en assises assez régulières, sont larges d'environ $70 \mathrm{~cm}$. Seuls les murs 4.102 et 4.127 sont dotés d'une ouverture (OUV 4.129 et OUV 4.145) dont les montants sont aménagés directement dans l'épaisseur des murs, tandis que la fondation sert de base pour un seuil, aujourd'hui 
disparu dans les deux cas. La porte 4.129 est large de $104 \mathrm{~cm}$, tandis que la porte 4.145 conserve uniquement son montant nord-est.

Fig. 3 - Mirine-Fulfinum, plan général des vestiges du secteur de «l'église à trois absides " (dessin D. Vuillermoz d'après $M$. Čaušević-Bully et $S$. Bully)

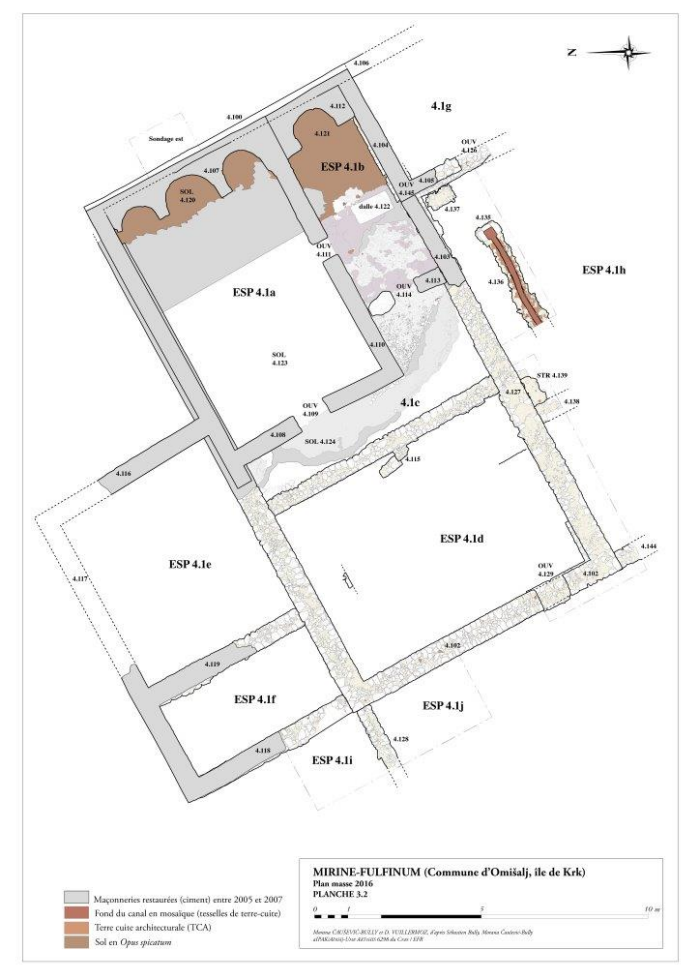

9 Un mur (4.115) de $56 \mathrm{~cm}$ d'épaisseur en moyenne, parallèle aux murs 4.100 et 4.102, est construit à l'intérieur de la partie principale, à 5,78 $\mathrm{m}$ de la façade maritime, créant ainsi deux espaces internes : Espace 4.1c, de $114 \mathrm{~m}^{2}$ dans la partie nord-ouest, et Espace $4.1 \mathrm{~d}$ de $66,5 \mathrm{~m}^{2}$ dans la partie sud-ouest. Ce mur a servi de soutènement de terrasse et à l'aménagement d'étages organisés en palier. En effet, la partie nord-est de l'espace central a été comblée, autorisant l'aménagement d'une pièce (Esp. 4.1c) dont le niveau de sol a été surélevé par rapport au niveau de circulation de la pièce aménagé du côté sud-ouest du MUR 4.115 (Esp. 4.1d). Cette dernière pièce a sans doute été surmontée d'un étage, dont le niveau de sol correspondait au niveau du sol de l'espace 4.1c. Ajoutons que l'espace 4.1c a été doté de plusieurs sols: un sol en opus spicatum (SOL 4.120) concentré dans la partie nord-est de la pièce et le sol 4.124, fin terrazzo, recouvert par un sol plus grossier dans une seconde phase (SOL 4.123). La limite très nette du sol 4.120, à environ 3,40 m du chevet, laisse supposer l'existence d'un autre mur de refend, aujourd'hui disparu. Ce "mur fantôme" diviserait l'espace 4.1c en deux ; il se situerait par projection dans le prolongement du mur 4.105 et du montant de la porte 4.145 .

10 La fouille du secteur 4.1c révèle un important remblai qui couvrait directement une épaisse couche de démolition du sol de l'étage. Cette couche du sol écroulé n'a pas été entièrement fouillée (prévue pour l'année 2017), cependant, l'abondant mobilier céramique en provenant nous donne d'ores et déjà des premiers éléments de datation de son abandon, qui ne dépasse pour l'instant pas le seuil du VI ${ }^{\mathrm{e}}$ siècle. 

l'espace $4.1 \mathrm{c}$, ce qui nous a permis de constater que le sol 4.124 recouvre un
comblement mis en place dès l'origine. Cela nous permet d'avancer l'hypothèse d'un
espace surélevé et construit en terrasse. du mur 4.102 et au sud du mur 4.127. Les murs appuyés contre les parements du bâtiment central sont d'une épaisseur moins importante. Le mur 4.128 , de $48 \mathrm{~cm}$ d'épaisseur, est ajouté à la façade 4.102, ses fondations épousent la forme des fondations de la façade, en reposant directement sur son talon (fig. 4). Ce mur reste isolé et aucun niveau de sol lié à son utilisation n'a été reconnu. Deux murs (4.144 et 4.138) accolés au mur 4.127, épais d'environ $53 \mathrm{~cm}$, créent des espaces distincts du côté sud. Le mur 4.144 est ajouté à l'angle extérieur formé par les murs 4.127 et 4.102, en prolongement de ce dernier, tandis que le mur 4.138, parallèle au mur 4.144, se situe à 5,10 m de la façade vers le nord-est. Une structure (4.139) préservée dans l'angle créé par les murs 4.127 et 4.138 pourrait correspondre aux vestiges d'un sol construits en bloc de pierre et mortier ou encore à une banquette appuyée contre le parement du mur 4.127.

Fig. 4 - Mirine-Fulfinum, détail du mur 4.102 et des murs 4.128 et 4.118 (cl. T. Chenal)

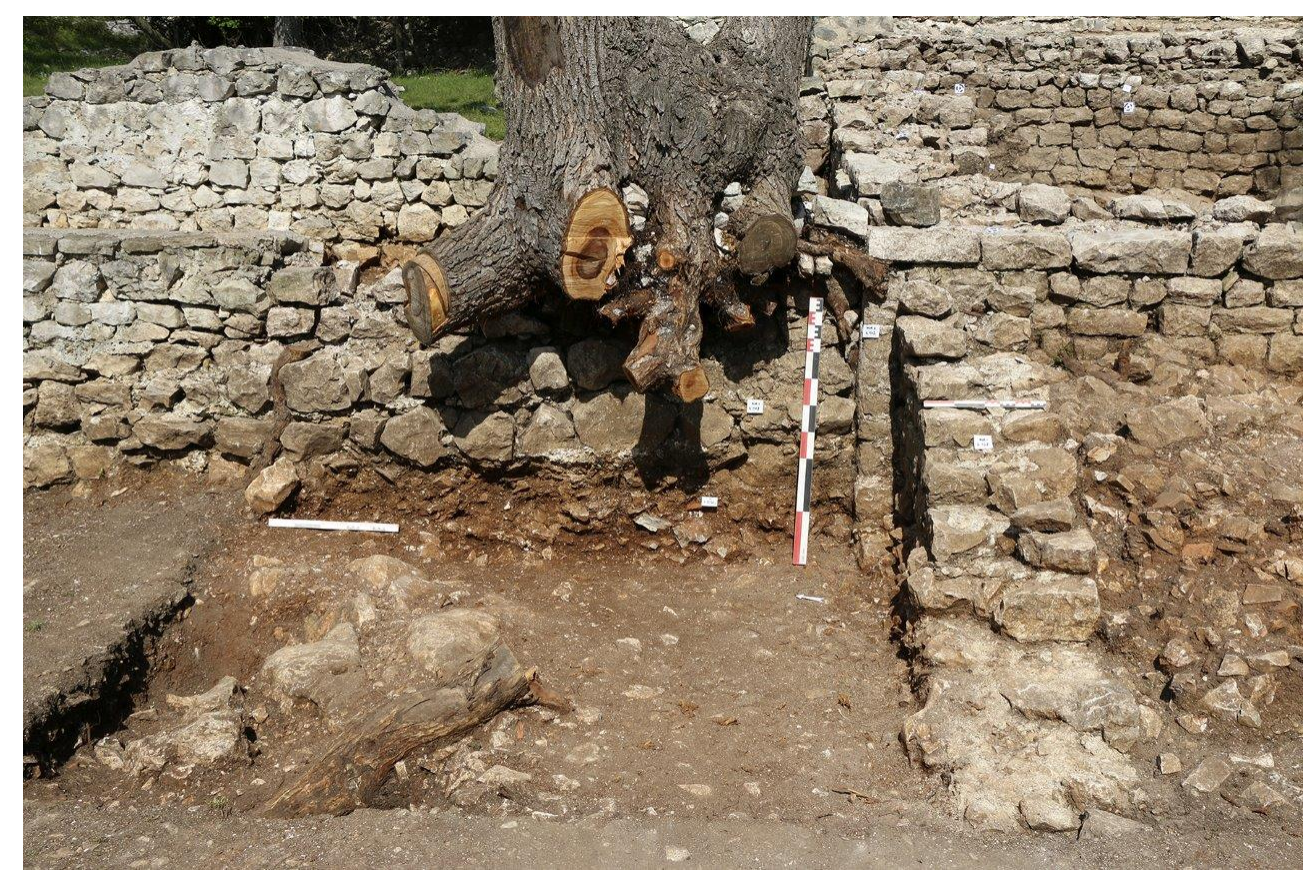

Plus au nord-est, la jonction des murs 4.127 et 4.105 est assurément contemporaine : le mur 4.105 est collé au parement du mur 4.127 dans sa partie basse, tandis que la partie haute surmonte le mur 4.127 et laisse la place à l'aménagement du montant oriental d'une ouverture (4.145), située ainsi en position quasi-centrale dans l'Esp. 4.1c. Le mur 4.105 possède une autre ouverture (4.126), située à la même côte que la porte 4.145. Ces deux ouvertures plaident d'avantage en faveur d'un niveau de circulation rehaussé dans les parties nord-est du complexe, ou plus précisément dans les espaces 4.1c et $4.1 \mathrm{~g}$ au nord-est du mur 4.105.

$14 \mathrm{Au}$ pied des murs 4.127 et 4.105, la fouille a révélé une structure (4.137) appuyée contre le mur 4.105, parementée du côté sud et sud-ouest, mais pas au contact avec le mur 
4.105. S'agit-il d'un aménagement dans l'angle de la pièce qui aurait pu supporter un niveau de circulation reliant les portes 4.145 et 4.126 ? La grande quantité de céramique mise au jour au pied de ce mur (US $4.1015 \mathrm{~h}$ ) présage peut-être d'une fonction culinaire et de l'existence d'un vide sanitaire au-dessous du niveau de circulation? La proximité du canal 4.136 doté d'un regard 4.135 plaiderait en faveur de cette dernière hypothèse (fig. 5). Cet aménagement hydraulique est d'une très belle facture: le regard est construit en appareil de moellons calcaire et ouvre sur le canal construit quant à lui en briques. Une tuile portant le timbre de l'atelier Q. Claudi Ambrosi est remployée dans sa construction (fig. 6). Presque tout le timbre est visible avec les lettres AMBROSI, dont les $\mathrm{AM}$ et SI sont des ligatures. Ces tuiles ont été produites dans la région aquilléenne $\mathrm{au} \mathrm{I}{ }^{\mathrm{er}}$ et dans la première moitié $\mathrm{du} \mathrm{II}^{\mathrm{e}}$ siècle $\mathrm{e}^{7}$. Le fond du regard et du canal est composé de tesselles en terre cuite. Le mobilier de ce secteur (4.1h) plaide en faveur pour une première phase d'occupation au $\mathrm{III}^{\mathrm{e}}$ siècle. La poursuite des études céramologiques devrait permettre de préciser les datations.

Fig. 5 - Mirine-Fulfinum, regard 4.135 et canal 4.136 avec son fond mosaïqué (cl. T. Chenal)

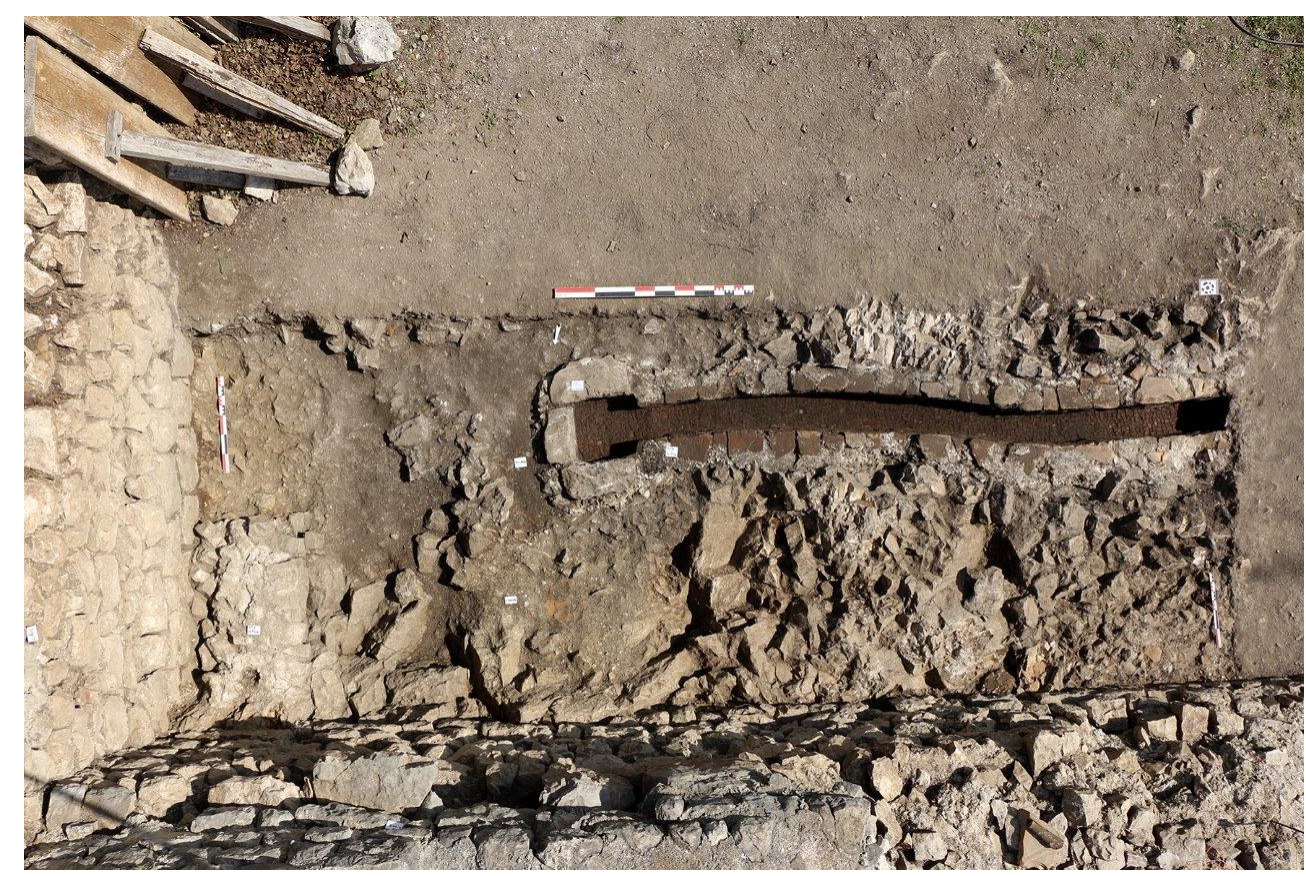


Fig. 6 - Mirine-Fulfinum, détail du canal 4.136 avec une tuile portant l'estampille AMBROSI (cl. M. Čaušević-Bully)



Un bâtiment rectangulaire, formé par les murs 4.116, 4.117 et 4.118, est ajouté au parement externe nord-ouest du mur 4.101. Un mur de refend (4.119) le divise en deux pièces : Espace 4.1e au sud-ouest, de $31 \mathrm{~m}^{2}$, et Espace $4.1 \mathrm{f}$ au nord-est de 13,6 $\mathrm{m}^{2}$. Les murs 4.116 et 4.117 n'ont pas pu être analysés lors de la campagne 2016, en raison de l'étendue limitée de la fouille et de leur restauration prématurée lors des travaux menés en 2005. Mais la lecture des maçonneries 4.118 et 4.119 et de la stratigraphie permet d'affiner leur chronologie relative avec les autres structures et d'avancer avec assurance que le mur 4.118 est postérieur aux murs 4.128 et 4.102 . L'aspect de la mise en œuvre de la maçonnerie du mur 4.119, avec ces blocs irréguliers et approximativement assisés, trouve son pendant avec le mur 4.118.

\section{Insertion de l'église à trois absides}

Le bâtiment central du complexe antique suburbain a subi d'autres remaniements importants. Alors que les salles $4.1 \mathrm{e}$ et $4.1 \mathrm{f}$ semblent s'appuyer contre un complexe toujours en fonction, la chapelle à trois absides et son annexe sud semblent reprendre des murs partiellement ruinés du bâtiment primitif. La chapelle (ESP 4.1a) prend place dans l'angle créé par les murs 4.100 et 4.101 , en empiétant avec son mur de chevet (4.107) sur l'arase de mur 4.100, aux deux tiers de son épaisseur, laissant les élévations du mur antique dépasser d'environ $18 \mathrm{~cm}$ du côté nord-est en guise de nouvelle fondation de la chapelle. Le chevet plat de la chapelle (4.107) comporte trois absidioles semi-circulaires inscrites, de forme irrégulière, avec des diamètres de 1,27 $\mathrm{m}$ pour l'absidiole nord, 1,76 m pour l'absidiole centrale et 1,35 pour l'absidiole sud (fig. 7). Le côté sud est constitué du mur 4.110 qui est doté d'une porte (4.111) permettant la communication avec la chapelle annexe (ESP 4.1b). La façade de la chapelle (4.108) se situe à $8,12 \mathrm{~m} \mathrm{du}$ fond de l'absidiole centrale ; elle possède une ouverture en position centrale (4.109). Le sol de la chapelle réutilise directement celui bâtiment antique : l' 
opus spicatum de la pièce I.4c délimite désormais le sanctuaire, tandis que la nef est couverte d'un simple sol en mortier de chaux.

Fig. 7 - Mirine-Fulfinum, vue du chevet à triple abside au moment des premières fouilles en 2005 (cl. M. Čaušević-Bully)

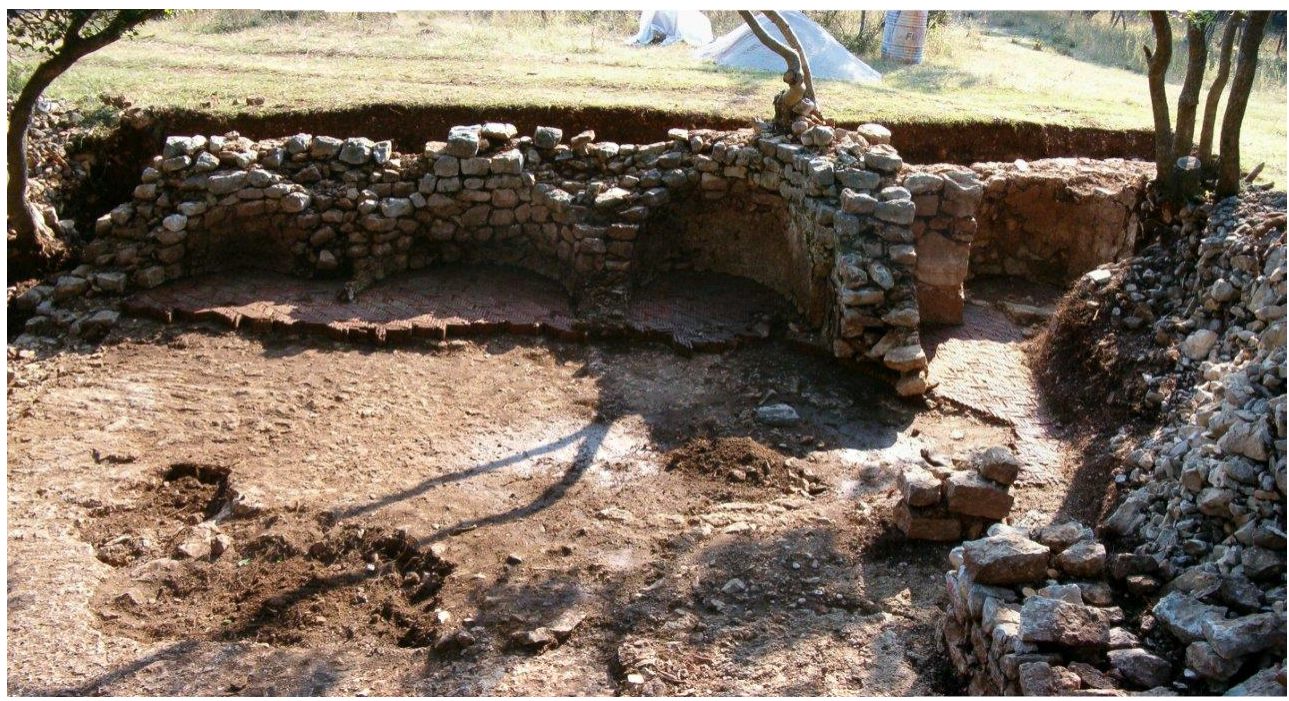

17 Un sondage ouvert au chevet du mur 4.100 a permis de constater l'absence de sépultures pouvant être liées à une éventuelle fonction funéraire de la chapelle. Ce sondage n'a par ailleurs révélé aucune structure ou niveau pouvant fournir des éléments de datation pour la construction de la chapelle. Aussi, une datation plus fine que la fourchette admise entre le $\mathrm{IX}^{\mathrm{e}}$ et le $\mathrm{XI}^{\mathrm{e}} \mathrm{s}$. pour la construction du petit complexe religieux fait toujours défaut.

18 Une chapelle annexe (Espace 4.1b) est ensuite greffée dans l'angle formé par les murs 4.100 et 4.103 , dans l'espace laissé libre par l'insertion de la chapelle à trois absides (Esp. 4.1a), d'une largeur de 2,46 m. Le chevet de la chapelle annexe (4.112) est construit dans le prolongement du chevet 4.107, en utilisant également le mur antérieur 4.100 comme fondation. Ce chevet plat comporte une absidiole inscrite de $1.32 \mathrm{~m}$ de diamètre. Notons qu'une ara antique est remployée pour former l'angle nord-ouest de l'absidiole. Au centre de l'espace de l'abside se dressait une base d'autel maçonnée (AUT 4.121), découverte en 2005, mais aujourd'hui entièrement disparue. Le Mur 4.111 de la chapelle 4.1a sert de mur nord-ouest à la chapelle annexe, tandis que son pendant sudest (MUR 4.104) est construit directement sur les arases des murs antiques 4.103 et 4.127 (fig. 8). Sa façade (4.113) est composée d'un mur construit directement sur le sol antérieur (4.123) et d'un long bloc de pierre en réemploi qui a servi de base pour la partie nord-ouest de la façade; la porte (4.114) se situe entre les deux parties, en position centrale. La façade est construite à $6 \mathrm{~m}$ de distance du fond de l'absidiole 4.112, et, par sa position, correspond à l'arrêt des fondations du mur 4.104, visibles au-dessus du mur 4.127 depuis le sud-est. 
Fig. 8 - Mirine-Fulfinum, détail du mur 4.101 de la chapelle haut médiévale construit sur l'arase du mur antique 4.127 (cl. T. Chenal)



\section{Sondage dans l'atrium de l'église de Mirine}

Durant la campagne de prospections géophysiques effectuée en 2012, nous avions engagé des prospections extensives dans le préau de l'atrium de l'église paléochrétienne de Mirine ( $c f$. supra). Rappelons brièvement que cet atrium fut rajouté au portique de l'église dans une seconde phase datée du $\mathrm{VI}^{\mathrm{e}}$ siècle si on se fie aux datations $\mathrm{C}^{14}$ réalisées sur les sépultures situées dans ses portiques. Les prospections géophysiques avaient révélé une structure, apparemment construite, située dans la partie sud-ouest du préau. Dès lors, nous avions suggéré plusieurs hypothèses d'interprétation, comme un mausolée ou encore une citerne.

Nous avons donc ouvert un sondage à l'emplacement de la structure présumée (fig. 9). Le sondage a été malheureusement négatif: les très nettes "maçonneries " reconnues par le radar-sol n'étaient rien d'autre que le comblement, avec un dense cailloutis, d'anciennes tranchées de sondages non documentées réalisées au début des années 2000. 
Fig. 9 - Mirine-Fulfinum, détail du canal 4.136 avec une tuile portant l'estampille AMBROSI (cl. M. Čaušević-Bully)

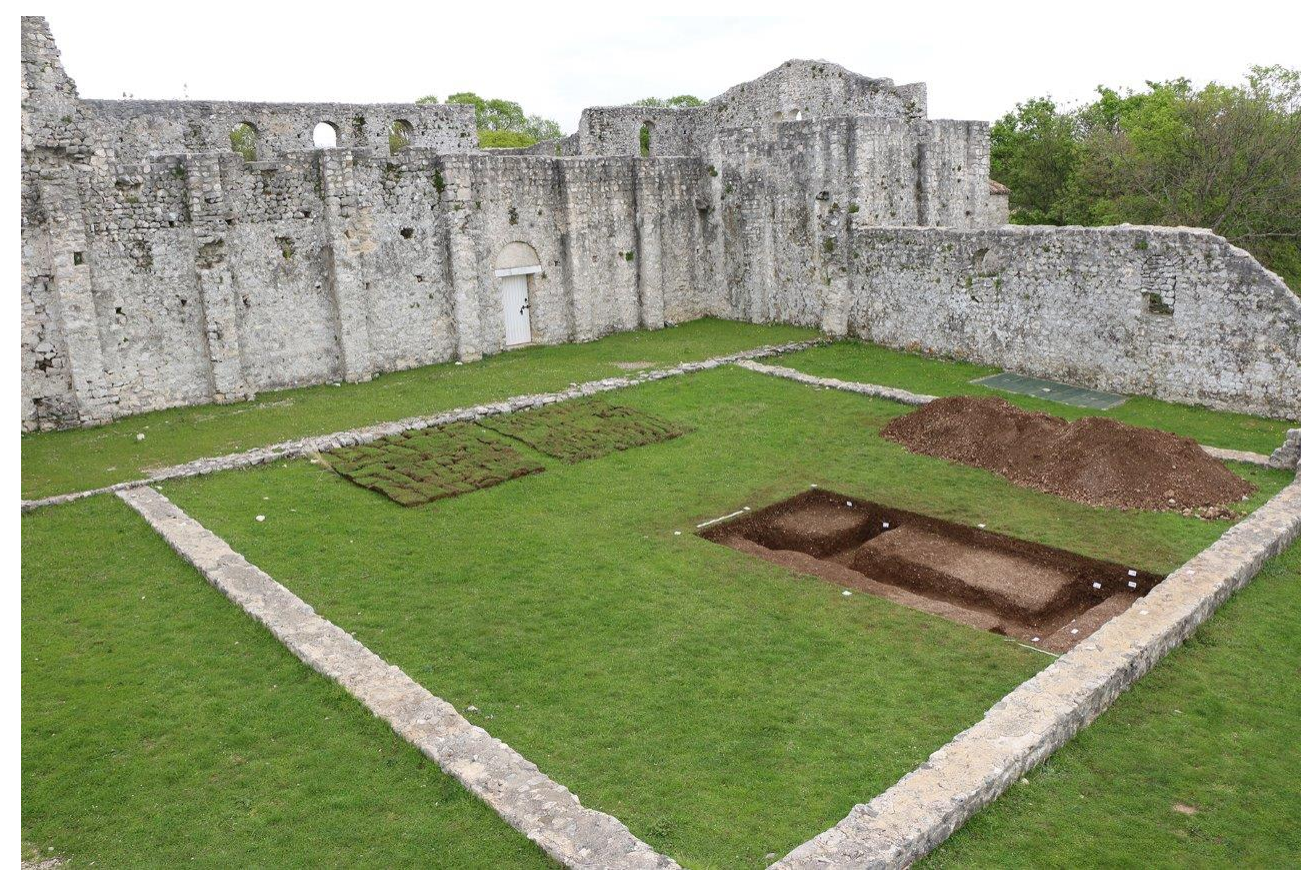

\section{Conclusion}

21 Le site du complexe antique suburbain présente un potentiel archéologique important. Cette première campagne de fouille nous permet d'ores et déjà d'avancer de nouvelles hypothèses quant à sa fonction et à sa chronologie (fig. 10). 
Fig. 10 - Mirine-Fulfinum, sondage dans l'atrium de Mirine (cl. D. Vuillermoz)

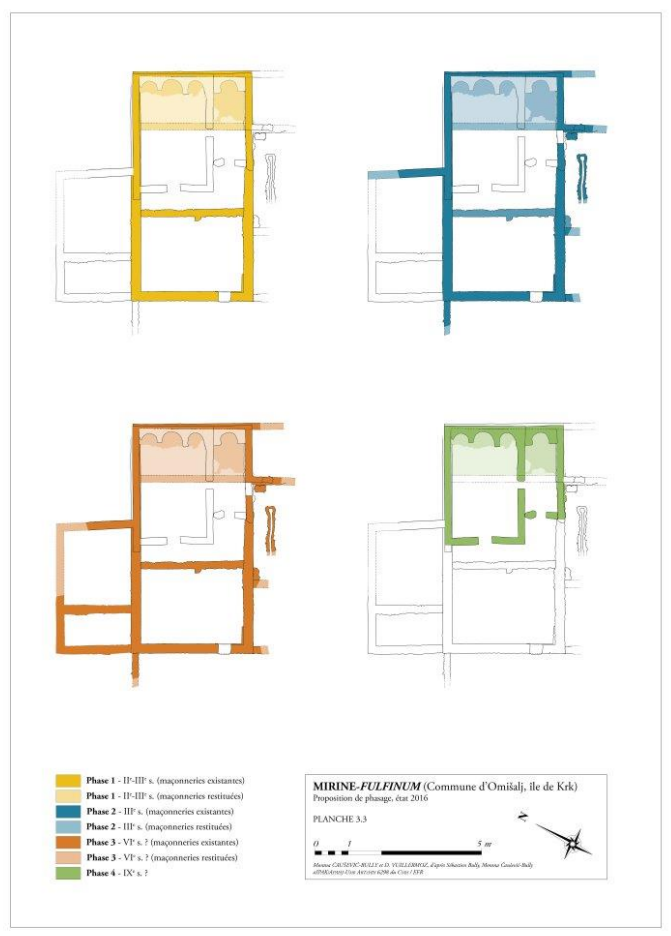

Dans l'état actuel des recherches, il apparaît que le petit complexe maritime suburbain aurait été établi sur les marges septentrionales de la ville de Fulfinum entre le $\mathrm{II}^{\mathrm{e}}$ et le $\mathrm{III}^{\mathrm{e}}$ siècle - d'après une première étude du mobilier céramique ${ }^{8}$. Ce complexe, dont l'étendue nous échappe pour l'heure, possédait une partie maritime, dotée d'un petit balnéaire et d'un corps de bâtiments aménagé sur deux étages en fonction de la déclivité naturelle du terrain. Les étages inférieurs - seulement au début de leurs fouilles -, semblent avoir été réservés à une fonction économique et domestique, comme en témoignent plusieurs blocs comportant des mortaises destinées à accueillir des éléments en bois d'un pressoir (à huile ?). La poursuite des recherches devrait apporter plus d'éléments sur ces aménagements. A contrario, les parties surplombant la mer étaient dotées de sols de bonne qualité (opus spicatum) et on peut présager une éventuelle fonction résidentielle, même si les éléments décisifs font encore défaut. La petite villa suburbaine a subi plusieurs remaniements et agrandissements tout au long d'une période d'occupation estimée jusqu'au $\mathrm{VI}^{\mathrm{e}} \mathrm{s}$. d'après une première analyse du mobilier céramique. Des pièces annexes (4.1c et 4.1d) - non encore fouillées - ont été ajoutées de part et d'autre du corps de bâtiment central.

Mais c'est manifestement dans un complexe abandonné et en partie ruiné qu'est construite l'église à trois absides (4.1a), accompagnée d'une chapelle annexe (4.1b). Les murs des deux petits édifices de culte sont en effet fondés sur l'arase des murs antiques; en revanche, il est plus surprenant que les sols antiques des parties surélevées aient été réutilisés comme sol de l'église et de sa chapelle latérale, comme s'ils étaient encore bien visibles. On ne peut cependant pas exclure que les travaux de terrassements pour la construction de l'église soient à l'origine de leur « découverte ». Quoi qu'il en soit, dans l'état actuel des recherches, rien ne permet de préciser la chronologie de l'église qui demeure comprise entre le $\mathrm{IX}^{\mathrm{e}}$ et le $\mathrm{XI}^{\mathrm{e}} \mathrm{s}$., d'après 
le type de plan. Et malheureusement aucune donnée n'accrédite l'hypothèse d'une fonction monastique, en l'absence de tombes - à ce jour -, comme de mobilier témoignant d'une occupation contemporaine de l'église. C'est vers l'interprétation d'une église votive, en lien avec sa situation dans la baie (pour des pécheurs, des marins?) que nous penchons désormais.

\section{Martinšćica, île de Cres (cinquième campagne) : fouille de la grande église (Martinšćica 1- deuxième campagne)}

Le complexe ecclésial de Martinšćica, situé sur le territoire de la cité antique et médiévale d'Osor, fait l'objet de recherches archéologiques dans le cadre du programme " Monachisme insulaire du $V^{e}$ au XI s. dans l'archipel du Kvarner » depuis 2011.

Les premiers résultats relèvent que Martinšćica est un site ecclésial majeur de l'archipel du Kvarner, eut égard à la singularité du parti architectural de sa grande église et des interrogations que suscite le complexe auquel elle appartient - une villa maritime antique et tardo-antique découverte lors des prospections en 2010. C'est la raison pour laquelle nous avions réalisé en 2014 la fouille de l'abside d'une salle de réception de l'Antiquité tardive de la villa maritime (Martinšćica 2), après un premier sondage en $2013^{9}$ (fig. 11).

Fig. 11 - Martinšćica, plan topographique général des vestiges (d'après M. Čaušević-Bully et S. Bully)

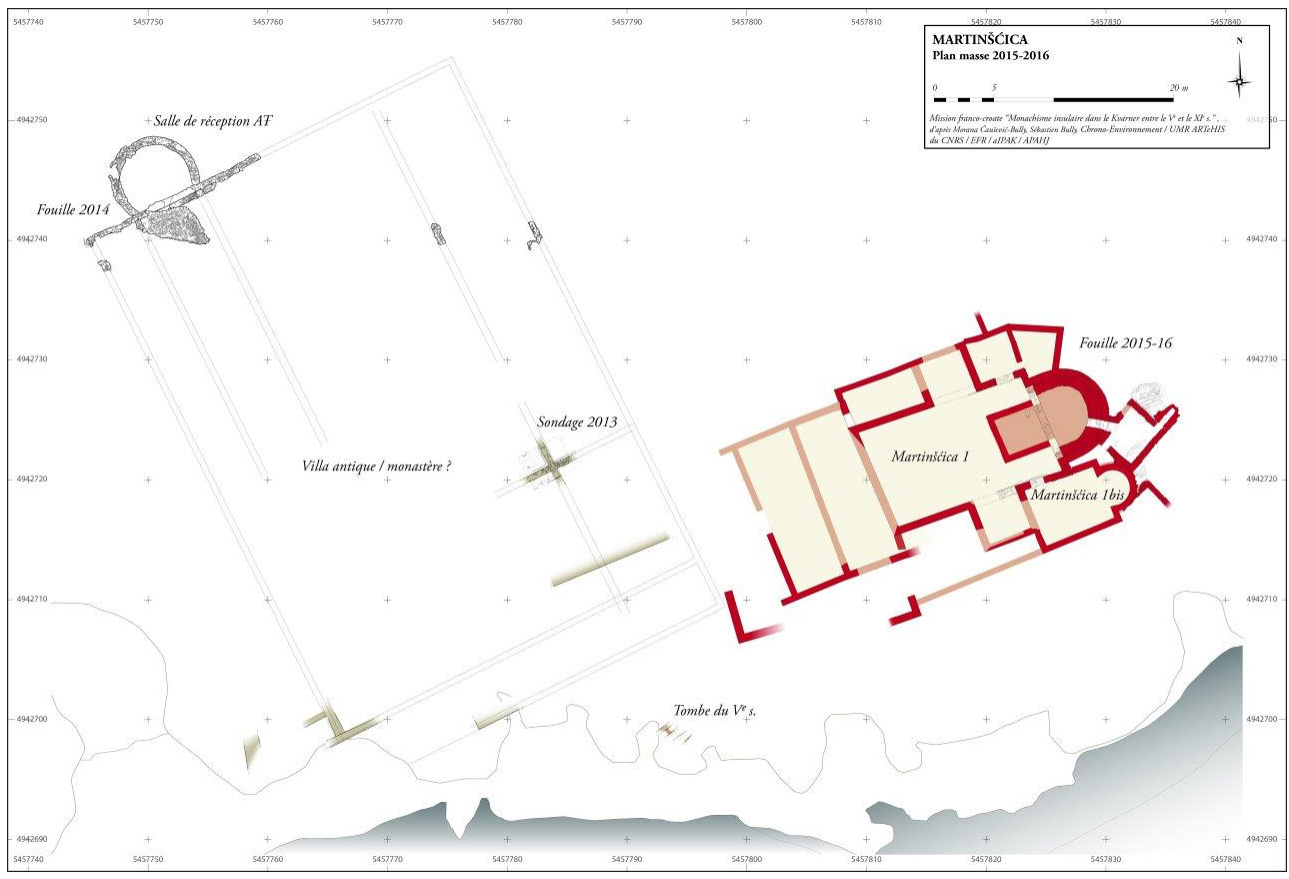

Les campagnes 2015 et 2016 ont porté sur la fouille de la grande église paléochrétienne (Martinšćica 1) au niveau de son abside (Ia), à son chevet (VIII), dans l'avant-chœur (Ib), dans une partie du bras nord du transept (Ic) et dans deux salles annexes au nord (sacristies IIIa et b) (fig. 12). 
La fouille de l'église doit permettre d'en définir le phasage et de comprendre la raison de la multiplication des annexes et leur fonction. Il s'agit également de comprendre les relations (?) entre le complexe résidentiel (villa) et le sanctuaire chrétien dans l'hypothèse de l'installation d'une communauté monastique.

Fig. 12 - Martinšćica, relevé pierre à pierre des parties orientales de la grande église (dessin F. Bully, d'après M. Čaušević-Bully et $S$. Bully)

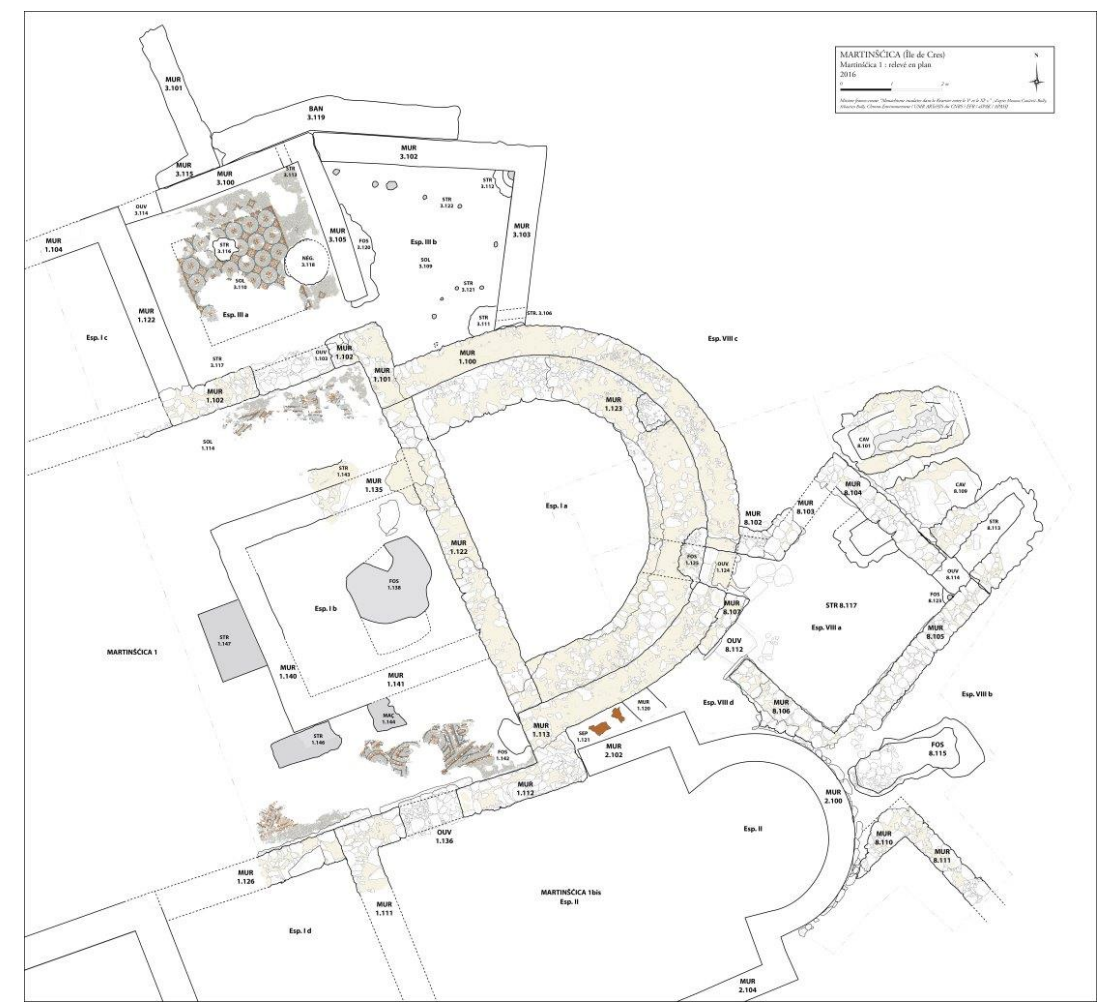

\section{Fouille de l'avant-choeur (Espace Ib)}

L'espace Ib, correspondant à l'avant-chœur, se situe entre le mur 1.122 - qui délimite l'espace de l'abside à l'est -, les épaulements de l'abside 1.101 et 1.113 , les murs gouttereaux 1.102 et 1.112 , et une limite - non matérialisée - donnée par l'extrémité orientale des bras du transept. Cet espace est en partie couvert d'un sol mosaïqué (1.114) dont on conserve de larges fragments contre le mur 1.102 au nord et le mur 1.112 au sud. La mosaïque est délimitée dans la partie centrale par les murs d'une plateforme de choeur (bêma), composée des murs 1.135 au nord, 1.141 au sud et 1.140 à l'ouest - préservé sur un segment de $163 \mathrm{~cm}$. Les trois murs mesurent $\pm 50 \mathrm{~cm}$ et délimitent une plate-forme de 4,52 x 4,12 m (dim. ext.). Les fragments de sculpture découverts nous assurent que la plate-forme était délimitée par une barrière de chancel.

La mosaïque 1.114 est endommagée par des fosses d'insertion de seuils tardifs : 1.131 pour l'insertion du seuil1.1027 de la porte 1.103 au nord et 1.132 pour l'insertion du seuil 1.1037 de la porte 1.136. Elle est également percée par la fosse 1.142 collée contre le mur 1.113. Le sol en mosaïque est couvert par les couches 1.1014 puis 1.1041, qui recouvrent directement les parties endommagées de sa couche de mortier 
préparatoire. Du côté sud, la couche de démolition 1.1044 - qui contenait notamment une colonnette en marbre-repose directement sur la mosaïque et le mortier du sol.

La plate-forme de chœur, située au centre de l'espace, débute immédiatement à l'est du transept et ménage deux passages latéraux, respectivement de 2,13 $\mathrm{m}$ le long du mur nord 1.102 et 2,03 $\mathrm{m}$ le long du mur sud 1.112. C'est dans ces deux passages, desservant à la fois la plate-forme par des ouvertures latérales et les annexes Ibis et IIIe qu'est le mieux préservé le sol de mosaïque.

Les vestiges d'un emmarchement maçonné (1.147) - 1,42 x 0,88 m - ont été identifiés au centre du "mur de façade» du podium. Deux autres structures latérales marquent deux accès supplémentaires: une première au nord (1.143) est visible à travers le négatif d'une marche $-38 \mathrm{~cm}$ de largeur par $97 \mathrm{~cm}$ de longueur maximale - reconnue dans le mortier du sol 1.114. Le second emmarchement au nord conserve partiellement une maçonnerie (1.143).

On observe un autre négatif de structure (1.146) dans l'angle sud-ouest de la plateforme - $51 \times 85 \mathrm{~cm}$. Le négatif porte les traces d'une structure qui aurait été dotée d'un placage (de marbre ?), dont les bords sont perceptibles dans le mortier du sol sur lequel la structure était posée. Cet aménagement de forme rectangulaire à angles coupés et accolé à l'angle du bêma pourrait appartenir à un ambon, auquel on accédait directement depuis le sanctuaire, en passant par la structure d'accès 1.144.

La plate-forme de chœur est très mal préservée, mais on note la présence d'une fosse (1.138) en position centrale (dimensions : 2,25 x 1,50 m) accolée au mur de la corde de l'abside (1.122) (fig. 13). Par sa position et la nature de son comblement, il est fort probable qu'il s'agit de l'arrachement d'une fosse d'autel que l'on suppose avoir été recouverte d'un placage de marbre d'après les fragments retrouvés. Le plan polymorphe de la fosse pourrait être le reflet d'un dispositif d'emmarchement coudé.

Fig. 13 - Martinšćica, vestiges de la plate-forme de chœur avec la fosse-reliquaire de l'autel comblée (cl. T. Chenal)

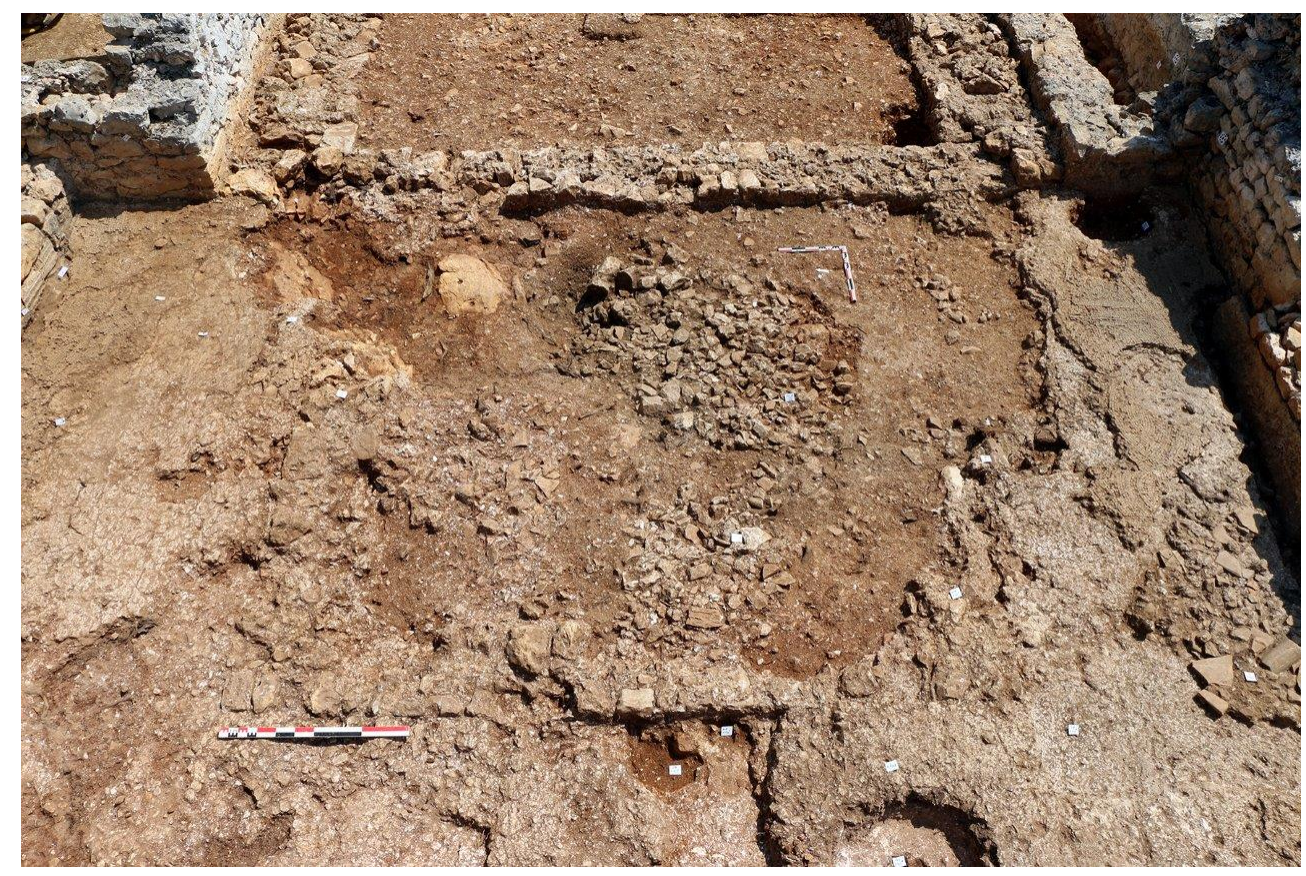




\title{
Fouille de la Sacristie IIla
}

34

\begin{abstract}
(1.122) et le mur gouttereau de l'avant-chœur (1.102). Les deux autres murs qui ferment la pièce, et qui sont chaînés, sont construits dans le prolongement des murs de l'église : le mur nord (3.100) est dans le prolongement du mur nord du transept (1.104) et le mur oriental (3.105) est dans le prolongement de l'épaulement nord de la grande abside (1.101).
\end{abstract}

35 La pièce IIIa est dotée d'une mosaïque polychrome (3.110), très proche de celle du sol 1.114 de l'église (fig. 14). Le champ décoratif central (2,15 x 2,15 m) est doté d'un seul motif de cercle qui se répète à l'intérieur du champ délimité par un trait noir puis rouge. Une structure hydraulique (3.113) a été aménagée dans le mur 3.100 au moment de sa construction. L'aménagement consiste en l'insertion de deux imbrices posées face à face pour créer un conduit de section presque circulaire. Les imbrices ont depuis disparu, mais leur négatif est toujours visible dans le mur. Le conduit a été fait pour évacuer l'eau à l'extérieur de la pièce. Nous noterons encore la découverte majeure dans le niveau d'abandon (3.1004) de cette salle d'un fragment de table d'autel en marbre comportant un monogramme - à ce jour non identifié - sur son revers (fig. 15).

Fig. 14 - Martinšćica, vue générale des sacristies Illa et IIlb depuis le nord-ouest (cl. T. Chenal)

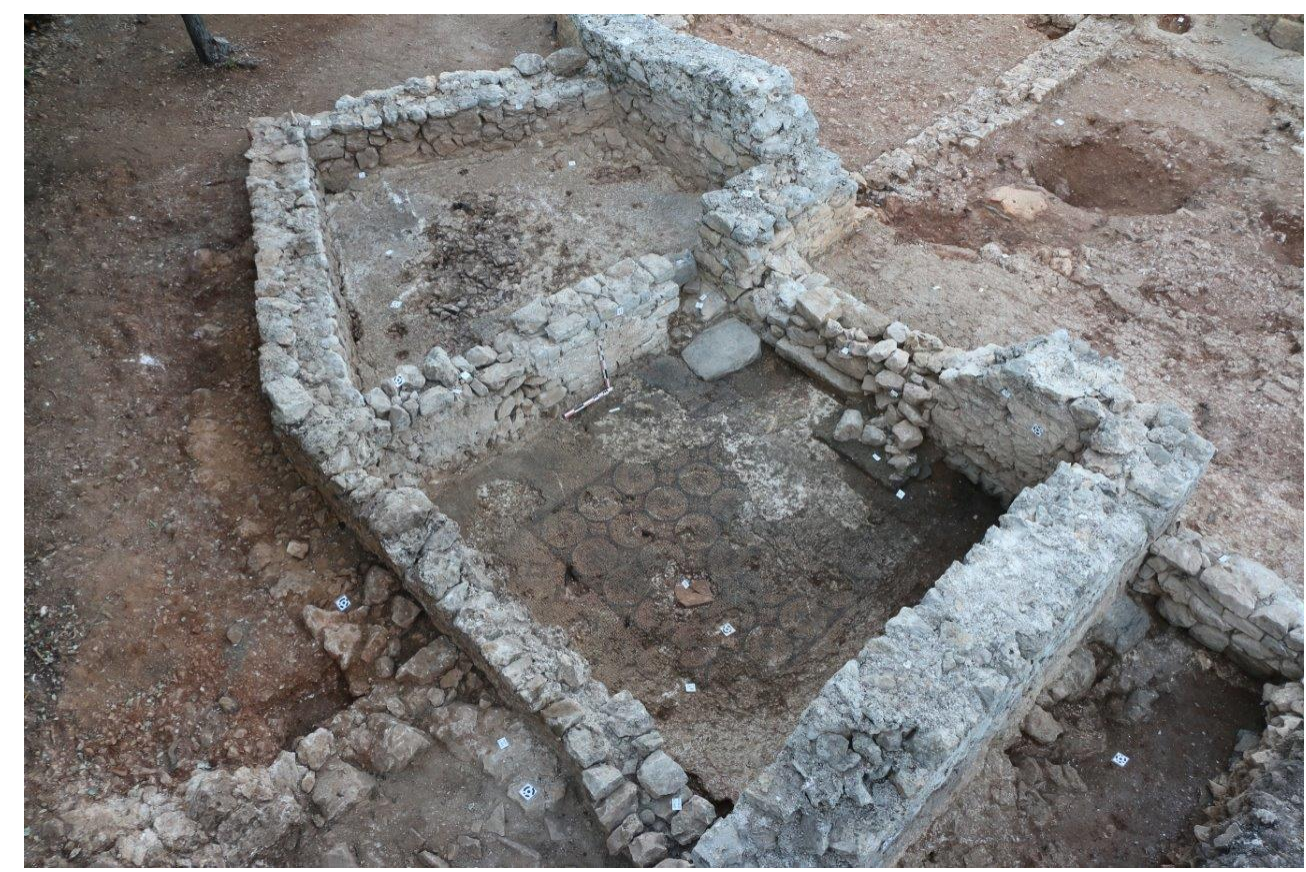


Fig. 15 - Martinšćica, revers de fragment de table d'autel en marbre avec monogramme (cl. J. Crochat)

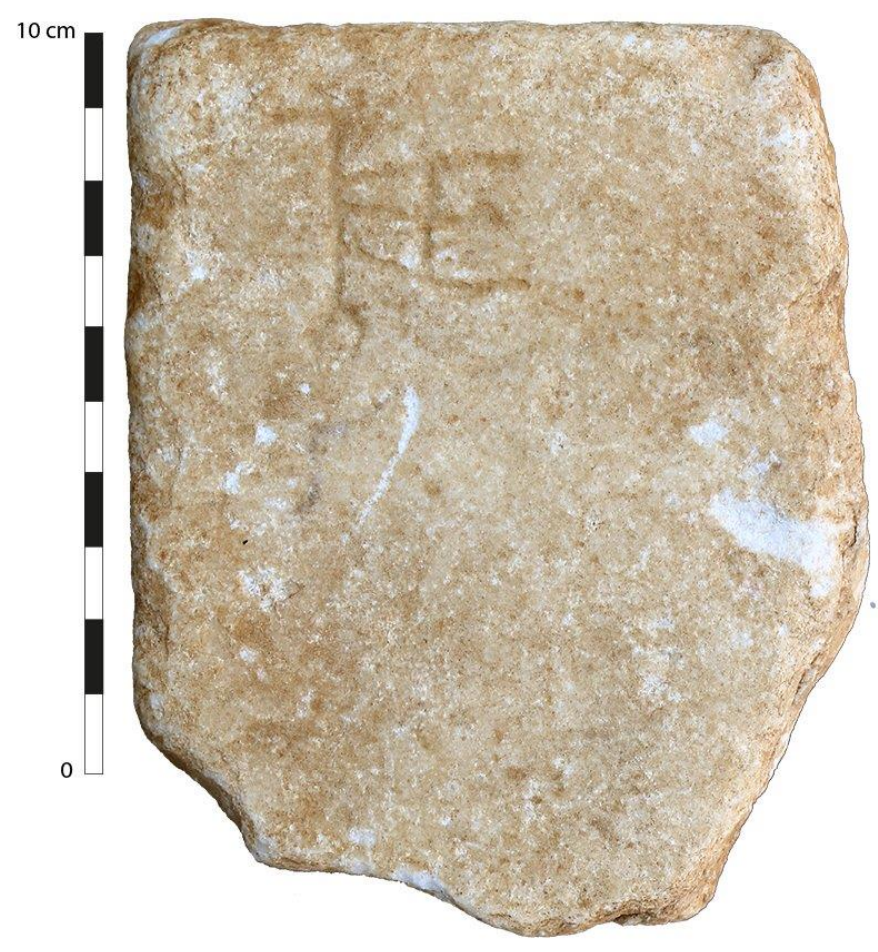

Dans une seconde phase, deux portes ont été percées dans les murs nord et est : la porte 3.114 (largueur : $78 \mathrm{~cm}$ ) et la porte $\mathbf{3 . 1 0 7}$ (largueur : 53,5 cm). Le montant nord de la porte 3.107 est construit en blocs de remplois, dont un fragment de barrière de chancel, et le mur 3.105 montrent une reprise importante des maçonneries correspondant à l'insertion de l'ouverture. Le sol a subi plusieurs phases d'utilisation. Dans un premier temps, il a été percé par l'aménagement d'une structure à la fonction indéterminée (3.116) ; dans un second temps, la mosaïque, fortement endommagée, a été réparée par une chape de mortier (US 3.1019), qui recouvre soigneusement les lacunes où manquaient les tesselles. Le négatif d'une importante structure circulaire (3.118) d'environ $85 \mathrm{~cm}$ de diamètre est visible dans cette couche de réparation, presque au contact avec le mur 3.105. Les réparations du sol sont ensuite scellées par une couche argileuse présentant les impacts thermiques d'un foyer (STR 3.117) situé dans l'angle des murs 1.122 et 1.102. Il est intéressant de noter que ce foyer est recouvert d'une couche cendreuse (US 3.1014), elle-même recouverte par l'obturation de la porte 1.103. Cette stratigraphie démontre que le foyer - et l'occupation contemporaine - est antérieure à l'abandon (désacralisation ?) de l'église, marqué par l'obturation de toutes les ouvertures.

\section{Fouille de l'Annexe IIIb}

L'espace IIIb est une annexe ajoutée à l'espace IIIa du côté est ; elle est donc délimitée par le mur 3.105 de l'annexe IIIa, puis par les murs 3.102 et 3.103 qui s'appuient, pour le premier contre l'angle formé par les murs 3.105 et 3.100, et pour le second, contre le parement extérieur de l'abside (1.100) (fig. 16). 
Fig. 16 - Martinšćica, détail du sol de la sacristie IIIb (cl. T. Chenal)

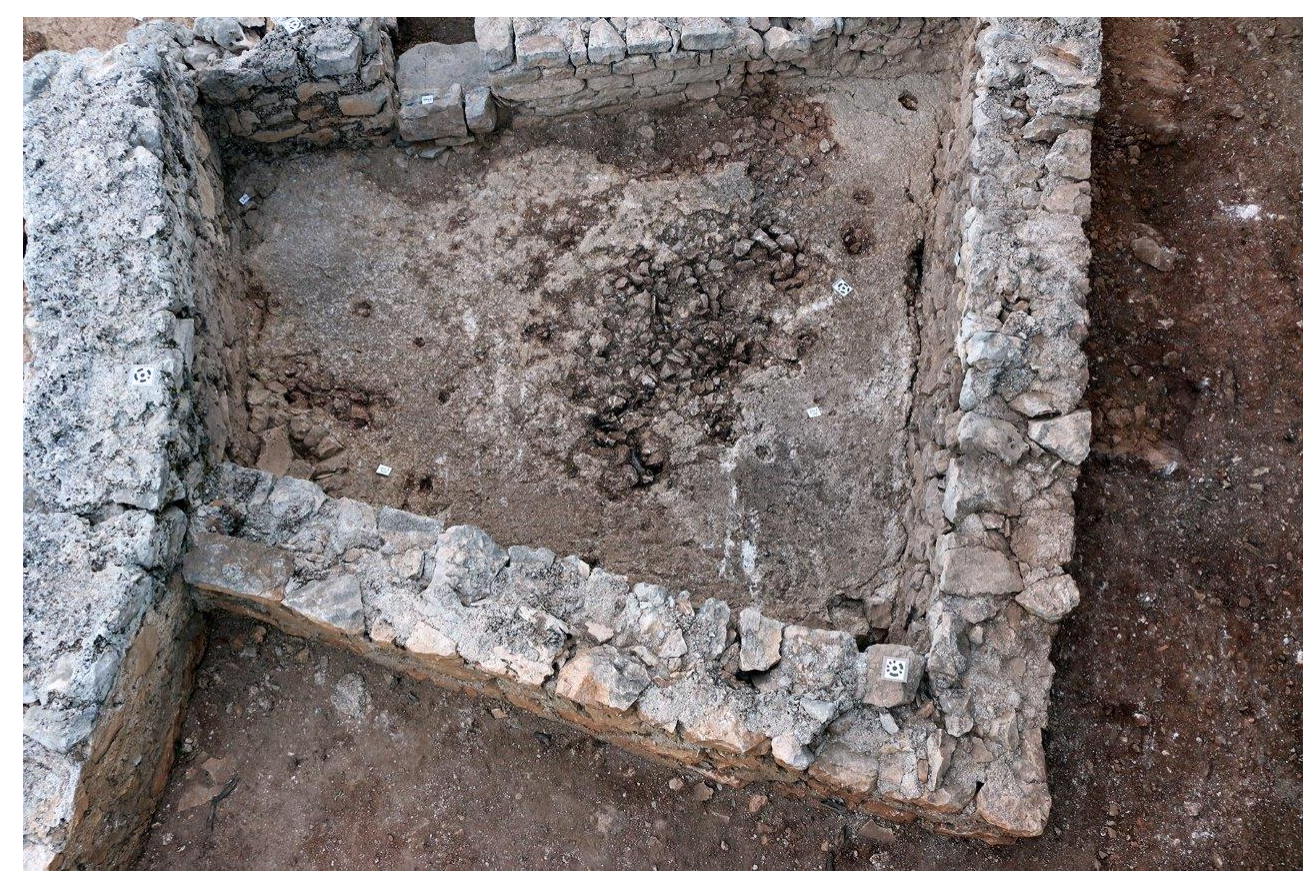

L'unique accès à cette annexe est assuré depuis l'espace IIIa par la porte 3.107 (cf. supra ). La pièce est dotée d'un simple sol en mortier de chaux (3.109), coulé directement sur un radier formé d'une couche de cailloutis très dense. Le sol conserve les vestiges d'un aménagement hydraulique (3.111) situé dans l'angle créé par les murs 3.103 et 1.100. Un cordon de blocs de pierre noyé dans le mortier dessine la base d'une structure organisée en quart de cercle; un grand fragment de cuve en calcaire, en position secondaire, occupe encore son centre. L'aménagement 3.111 fonctionne avec la canalisation 3.106 aménagée dans le mur 3.103 au moment de sa construction. Cet aménagement est de même type que la STR 3.113 de l'ESP. IIIa, et, comme lui, sert à l'évacuation de l'eau. Un second aménagement hydraulique (3.112) se situe dans l'angle des murs 3.102 et 3.103 ; il est également contemporain des murs et du sol. Les trois structures $(3.106,3.111,3.112)$ sont contemporaines et de la contemporanéité des murs de l'annexe avec le sol 3.109 .

Ce sol conserve les traces d'aménagements ultérieurs. Une première série de cinq trous de piquets, percés dans le sol (STR 3.121) et disposés en quart de cercle, souligne le piétement du lavabo 3.111 et devait supporter une table d'angle ou un large lavabo. Une deuxième série de six trous de piquets ou de petits poteaux (STR 3.122) se développe sur une ligne bordant le mur nord 3.102. Une fosse, probablement une tranchée (FOS 3.120) perce le sol 3.109, et se situe contre le mur 3.105. Cette fosse pourrait être liée à la reprise du mur 3.105, que nous avons évoqué plus haut.

\section{Fouille de l'Annexe VIIla}

L'espace VIIIa, situé au chevet de l'abside 1.100, a été en grande partie fouillé lors de la campagne 2015. Rappelons que cette annexe est composée des murs 8.103, 8.104, 8.105 et 8.106 ; les murs 8.102 et 8.107 assurent la jonction avec le parement externe de l'abside 1.100, où l'accès direct à l'espace du sanctuaire est rendu possible par la porte percée dans l'épaisseur du mur 1.100 (OUV 1.124). Un petit passage aménagé entre les 
murs 8.107 et 8.106 assure la communication entre l'annexe VIIIa et le petit espace VIIId (cf. infra).

La fouille de cette année a permis de mieux cerner les relations stratigraphiques et de comprendre la terminaison orientale de la pièce. Les vestiges très mal conservés du niveau de circulation (terre battue et mortier) contemporain de l'annexe ont été traversés afin de comprendre la stratigraphie antérieure. Nous avons ainsi pu constater que les deux tombes maçonnées (8.101 et 8.109), découvertes l'année dernière, situées en partie sous le mur 8.104, sont recouvertes par une aire de gâchage du mortier (8.117). L'aire de gâchage 8.117 est assurément antérieure de l'annexe VIIIa, car elle est scellée par plusieurs couches de remblai (US 8.1015/1025, puis 8.1014/1024) sur lesquelles s'installent les murs de l'annexe (fig. 17).

Fig. 17 - Martinšćica, vue générale de l'annexe VIIla (cl. S. Bully)

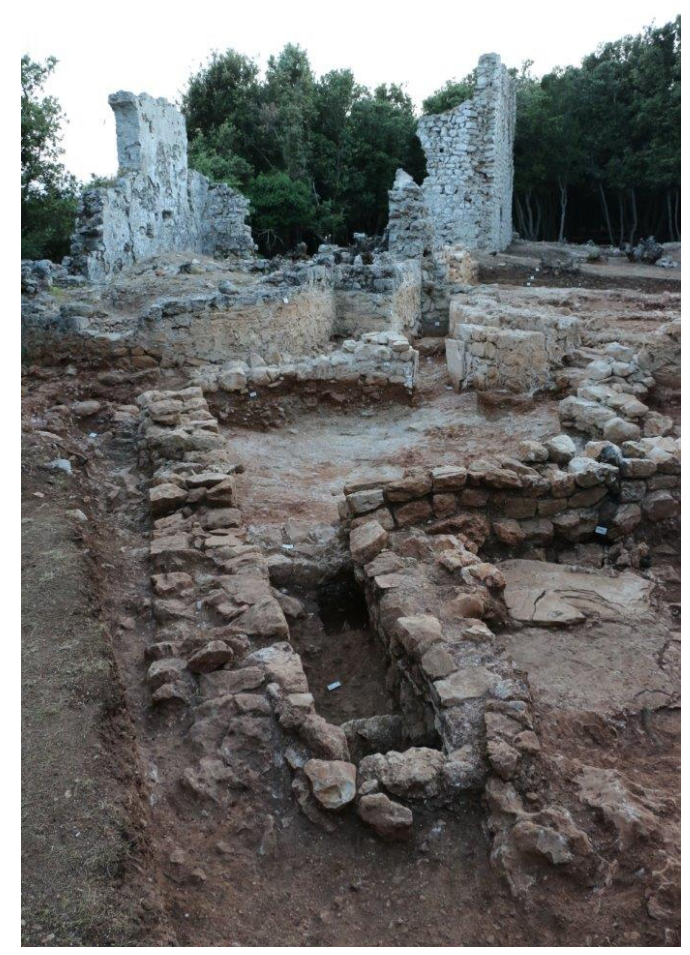

42 L'aire de gâchage s'étend au sud-est de l'abside et les couches de chaux encore préservées remontent contre les murs de l'abside. Par les relations stratigraphiques de cette structure avec les murs environnants, on peut conclure qu'elle a dû être créée au moment de la construction de la chapelle annexe Martinšćica 1bis.

Les fouilles ont été étendues également sur l'extrémité est de l'annexe, dans le prolongement de l'ouverture 8.114 et des murs 8.108 et 8.113 découverts l'an dernier. Les deux murs se rejoignent à $1,97 \mathrm{~m}$ du mur 8.104 (et l'OUV. 8.114), créant ainsi une unique structure $\mathbf{- 8 . 1 1 3}$ d'une largeur moyenne de $46 \mathrm{~cm}$. On pouvait accéder à la structure depuis l'annexe VIIIa par l'ouverture 8.114. La taille et la position de la structure correspondaient parfaitement à une tombe maçonnée, ce qui était l'hypothèse privilégiée. Mais contrairement à nos attentes, la fouille a démontré qu'il ne s'agit en aucun cas d'une sépulture. L'intérieur de la structure, entièrement fouillé, a seulement livré une dalle dressée de chant, légèrement en biais, compartimentant la 
structure à son extrémité orientale (fig. 18). Le court caisson était couvert de dalles uniquement à son extrémité. Le comblement (US 8.1030) contenait exclusivement des ossements de faune (probablement de la famille des Caprinae). Un petit pot, entièrement préservé (US 8.1033/objet 2016.01), a été déposé dans une petite fosse - creusée dans la STR 8.117-, située contre l'angle interne créé par l'ouverture 8.114 et le mur 8.105 (fig. 19). Le pot semble être en lien direct avec l'aménagement 8.113 et devrait fournir un indice de datation de la dernière phase d'utilisation de l'annexe VIIIa - et ces parois seront analysées à la recherche d'indices quant à son contenu. Ces découvertes plaident en faveur d'une occupation plutôt domestique de l'annexe, mais ne sont pas déterminantes, en l'absence d'autres structures - de type foyer - et de niveaux d'occupation démonstratifs - par le mobilier contenu.

Fig. 18 - Martinšćica, détail de la structure 8.113 de l'annexe VIIla (cl. T. Chenal)

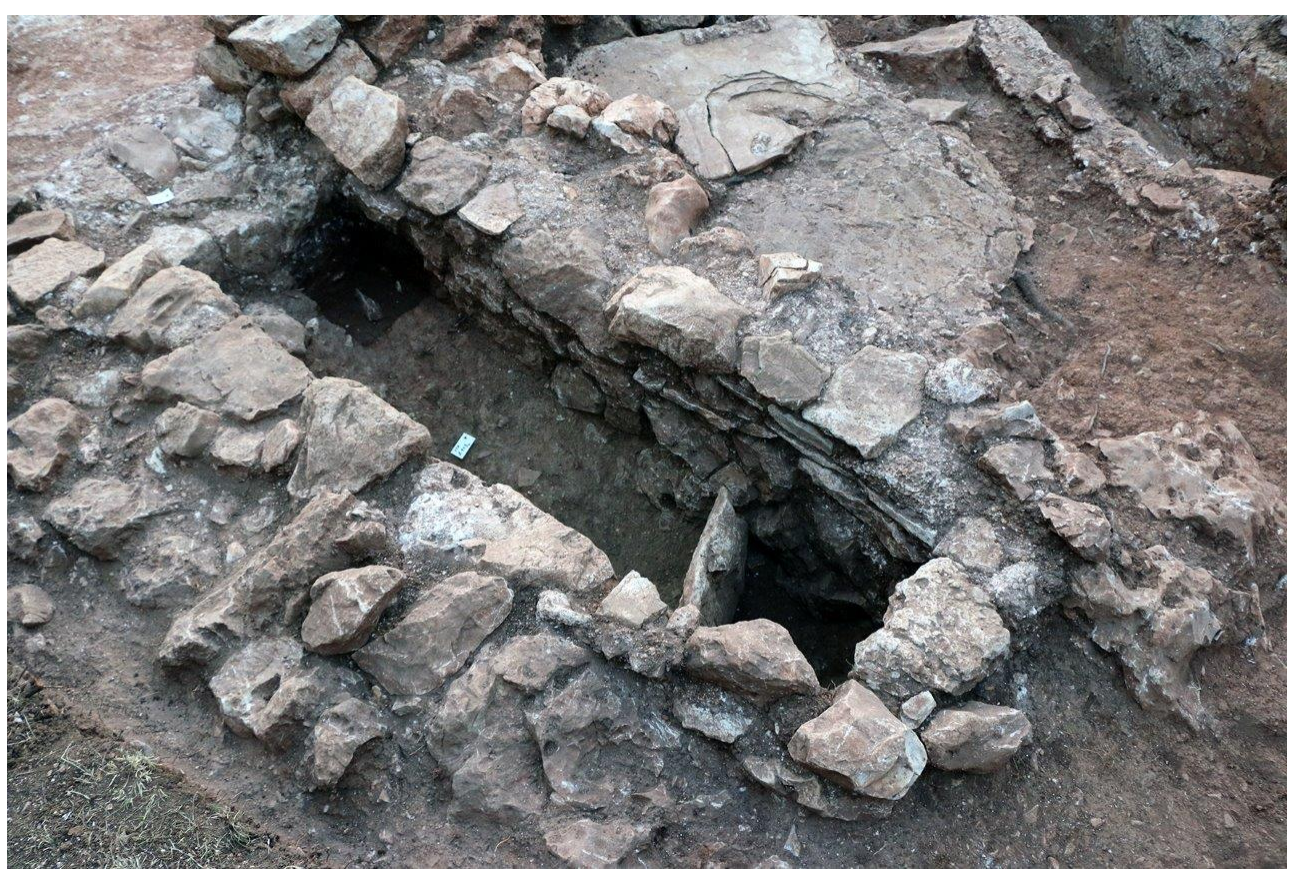


Fig. 19 - Martinšćica, détail du pichet en céramique déposé devant l'ouverture de la structure 8.113 (cl. J. Crochat)

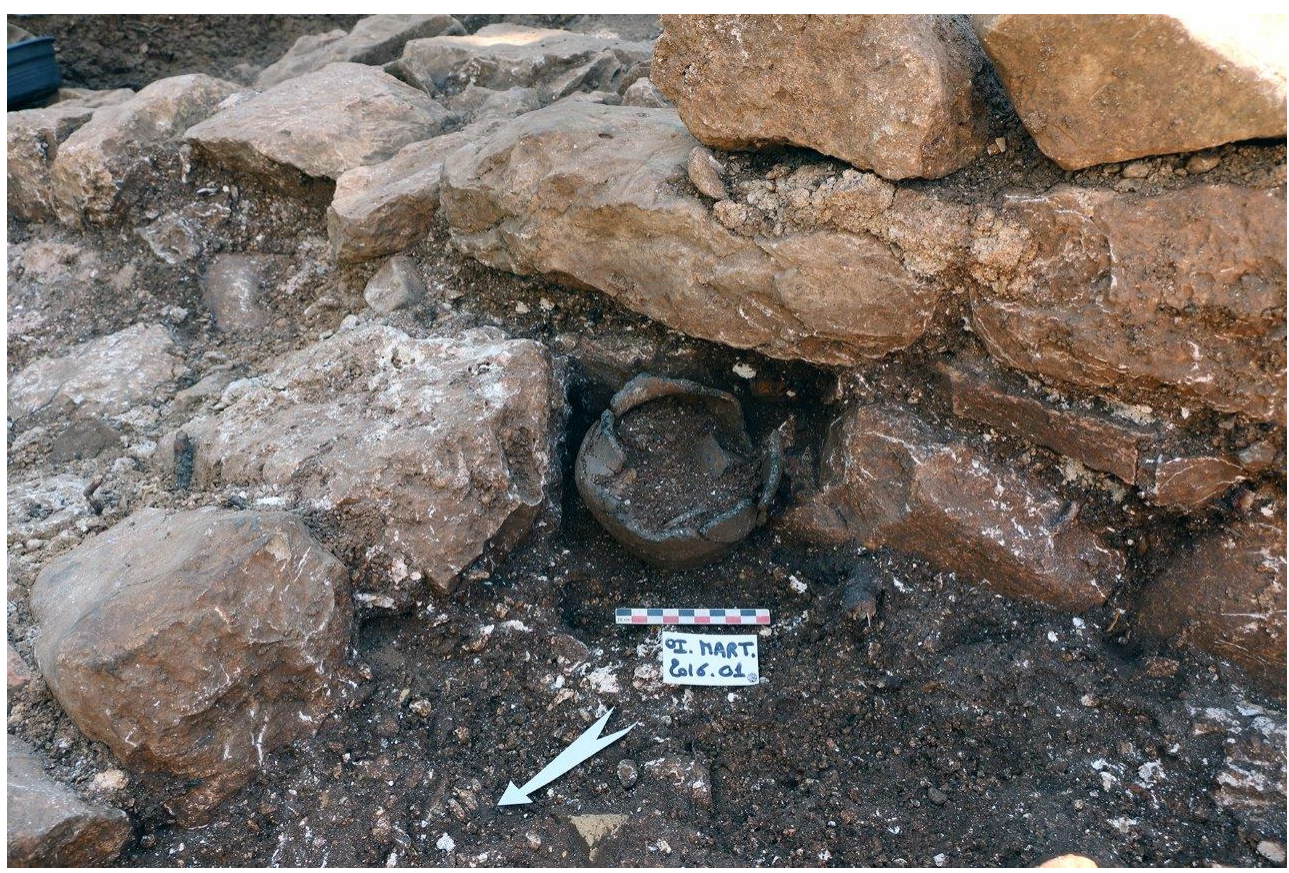

\section{Fouille de l'espace VIIId}

L'espace VIIId est délimité par les murs de la grande église (abside 1.100 et épaulement 1.113), de la chapelle annexe (2.102 et 2.100) et de l'annexe VIIIa (8.106). L'unique accès à ce petit espace est assuré par l'ouverture 8.112 aménagée entre les murs 8.106 et 8.107 de l'annexe VIIIa (fig. 20). 
Fig. 20 - Martinšćica, vue générale de l'annexe VIIId (cl. T. Chenal)



La fouille a permis de découvrir la continuité de l'aire de gâchage 8.117 qui recouvrait une maçonnerie plus ancienne (8.120) orientée nord-sud, dont le parement occidental se situe à $133 \mathrm{~cm}$ du parement externe de l'épaulement 1.113 pour son extrémité nord, et à $150 \mathrm{~cm}$ pour son extrémité sud. Cette maçonnerie, coupée par le mur 2.102 de la chapelle sud, suit une inclinaison par rapport à l'orientation des murs nord-sud de l'église. Elle appartenait sans doute à une annexe antérieure à la chapelle Martinšćica 1bis, mais pourrait également constituer une sorte de petite chambre funéraire. En effet, une tombe de bébé en amphore (SEP 8.121, Ind.3) a été également découverte près de l'épaulement 1.113. La stratigraphie dans ce secteur ne nous a permis de déterminer si elle a été déposée après ou avant la construction de la chapelle annexe. Il est en revanche très probable que ce fut avant la construction de l'annexe VIIIa. Extrêmement détériorée, l'amphore est difficile à identifier.

\section{Le pavement de mosaïque de la sacristie IIla (Pascale Chevalier)}

Le sol de l'annexe quadrangulaire (3,50 x 3,30 m environ dans l'œuvre) IIIa venue occuper le quadrant nord-est entre le bras septentrional du transept et le mur nord de la nef et dont le mur oriental s'aligne avec l'épaulement de la nef, est pavé d'un opus tesselatum polychrome à fond blanc et motifs noirs, blancs et rouges ${ }^{10}$ (cf. fig. 12 et 14). Cette sacristie, peut-être ajoutée dans un second temps, était accessible depuis le sud, par une porte ouvrant sur l'espace également mosaïqué qui s'étend au nord de la plateforme du chœur. Bien préservé sur environ un tiers de sa surface originelle, le tapis de mosaïque est fortement endommagé par endroits : seuls neuf des carrés sur la pointe de la bordure et 26 des 36 cercles du champ sont conservés, certains très partiellement. 
Encadré d'une très large bordure blanche ( $60 \mathrm{~cm}$ en moyenne), semée d'une file espacée de carrés sur la pointe dessinés en noir, et limitée intérieurement par un filet rouge et un filet noir, le champ central est un carré de 2,10 m de côté. Son décor consiste en une grille géométrique de trente-six cercles noirs (diam. $33 \mathrm{~cm}$ soit un pied), tangents mutuellement et avec le filet noir de la bordure, et disposés en six rangées de six. Chaque cercle est timbré d'un fleuron à croix, de forme arrondie, qui arbore un cœur fait d'une tesselle noire et quatre pétales rouges évasés à racine noire - le tout tendant à évoquer une petite croix très pattée. Dessinés régulièrement d'un trait en simple filet de tesselles noires, les trente-six cercles dégagent le long de la bordure des écoinçons triangulaires concaves et, entre eux, des carrés sur la pointe également concaves. Ces derniers contiennent un motif proche de celui qui timbre les cercles et identique à celui que contiennent les carrés sur la pointe de la bordure, mais en alternance de teintes et basculé en X. Il s'agit en effet de fleurons à croix de forme arrondie, avec pour ceux du champ central une petite croix de saint André rouge marquée d'une tesselle blanche centrale sur un fond blanc; tandis que dans les carrés sur la pointe de la bordure la petite croix blanche est marquée d'une tesselle noire centrale sur un fond rouge.

Les fleurons à croix d'aspect géométrique sont extrêmement répandus dans tout le monde romain tardif (ainsi à la villa de Pisoes près de Beja au Portugal au IV ${ }^{e}$ siècle ou à celle de Loupian en France au $\mathrm{V}^{\mathrm{e}}$ siècle) et difficiles de ce fait à dater avec précision. Dans la gamme de couleur de ceux qui timbrent les cercles, chaque fleuron réunit en croix quatre boutons de rose stylisés, liés par la tesselle noire centrale ; cet agencement cruciforme de boutons de rose rouges et les semis couvrants des mêmes boutons floraux sont assez caractéristiques des pavements du VI siècle, notamment en Syrie-Palestine. On en trouve d'ailleurs de nombreux exemples datés entre la fin du IV ${ }^{\mathrm{e}}$ et le $\mathrm{VI}^{\mathrm{e}}$ siècle, plus proches géographiquement de Martinšćica: à Poreč, sous une inscription dédicatoire des années $390^{11}$; à Novalja sur l'île de Rab, sur un tapis de mosaïque de l'abside de la grande basilique urbaine du début du V $\mathrm{V}^{\mathrm{e}}$ sècle $^{12}$; à Betika (certains dans des cercles en filet noir) sur le pavement de la memoria triconque de la première moitié du $\mathrm{V}^{\mathrm{e}}$ siècle ${ }^{13}$; à Pula dans le presbyterium de la cathédrale au $\mathrm{V}^{\mathrm{e}}$ siècle $^{14}$ ; à Ližnjan, dans la basilique paléochrétienne du $\mathrm{V}^{\mathrm{e}}$ siècle $^{15}$; à Salone (Basilica urbana, Marusinac) au V $\mathrm{V}^{\mathrm{e}}$ siècle ${ }^{16}$; à Saint-Jean l'évangéliste de Rab au V $\mathrm{V}^{\mathrm{e}}$-début $\mathrm{VI}^{\mathrm{e}}$ siècle $^{17}$; au cathecumeneum du baptistère de Salone dans la première moitié $\mathrm{du} \mathrm{VI}^{\mathrm{e}}$ siècle ${ }^{18}$; enfin à Poreč, dans un losange d'un panneau découpé provenant d'un sol non situé du complexe euphrasien (mi-VI siècle) ${ }^{19}$ et sur deux tapis du collatéral sud de la basilique euphrasienne ${ }^{20}$. Quelques tapis des $\mathrm{V}^{\mathrm{e}}$ et $\mathrm{VI}^{\mathrm{e}}$ siècles observés sur les sites déjà évoqués présentent des compositions géométriques tracées à l'aide de simples filets noirs, mais plutôt à bases de croix ou d'octogones imbriqués, comme à Novalja ; régionalement les systèmes à base de cercles jointifs générant des carrés concaves sont plus souvent dessinés par des tresses ou des tores cordés. Il n'existe donc pas de comparaison exacte.

Ici, c'est donc la chronologie relative des composantes de l'édifice qui permet d'avancer une datation $\mathrm{du} \mathrm{VI}^{\mathrm{e}}$ siècle pour ce pavement de mosaïque.

\section{Le mobilier lapidaire (Jessy Crochat)}

Les deux premières campagnes de fouille de l'église de Martinšćica I ont mis au jour 58 éléments lapidaires localisés dans les deux annexes nord (Esp. IIIa et IIIb), l'annexe du 
chevet (Esp VIIIa) ainsi que dans l'avant-chœur et le chœur de l'église (Esp Ia et Ib.) (fig. 21).

Fig. 21 - Martinšćica, localisation des blocs lapidaires découverts en 2015 et 2016 (d'après J. Crochat)



51 La plupart des blocs ont été découverts dans les niveaux de démolition des murs des annexes, suggérant qu'ils se trouvaient en remploi lors des phases tardives de l'église. En revanche, certains éléments peuvent assurément être rattachés à des structures fouillées, à l'image des fragments de placage en marbre.

Le mobilier lapidaire se répartit en trois groupes fonctionnels: les installations liturgiques, la sculpture architecturale et les éléments divers / indéterminés.

\section{La sculpture architecturale}

La sculpture architecturale se concentre au niveau des ouvertures, portes et fenêtres. Trois fragments appartenaient certainement au même transenne ou à un ensemble homogène de transennes au motif d'écailles de poisson. Un linteau découvert remployé en tant que seuil de l'ouverture 1.103 - est sculpté d'un chrisme excentré et d'un jeu de moulures en « dents de scie ». Peut-être utilisé pour cette même porte, nous avons découvert un fragment de montant sculpté d'un rinceau ondulé encadré également par un jeu de moulures. Toutefois, les éléments les plus abondants de sculpture architecturale sont concentrés au niveau du sol. On recense ainsi quelques probables fragments de seuil - ou de stylobates de chancel - ainsi que plusieurs fragments de dalles dont certains présentent un poli d'usure. 


\section{Le mobilier liturgique}

La fouille du chœur et de l'avant-choeur, associée aux éléments lapidaire découverts, a permis d'affiner notre connaissance de son organisation liturgique ainsi que de celle des annexes. Nous connaissons d'ores et déjà deux tables d'autel en marbre blanc pour Martinscica I: une mensa circulaire - ou semi-circulaire - moulurée d'inspiration antique et une table rectangulaire dont les tranches décorées d'une gorge peu profonde terminée dans les angles par des lunules sont particulièrement courantes durant l'antiquité tardive ( $\mathrm{IV}_{-} \mathrm{VI}^{\mathrm{e}}$ s.) (fig. 22). La découverte majeure, ici, réside dans l'inscription du monogramme précédemment écoqué. Bien qu'il ne soit pas possible de replacer ces deux tables d'autel dans l'espace, il n'est pas impossible qu'elles aient coexisté, l'une servant de table eucharistique, l'autre servant de table d'autel à la chapelle (esp. II).

Fig. 22 - Martinšćica, fragment de mensa en marbre (cl. et dessin J. Crochat)



Dans le chœur liturgique, l'autel était associé à une fosse reliquaire dont la campagne de fouilles 2016 a mis en évidence l'arrachement. Sept fragments de placage découvert dans le comblement de la fosse (FOS 1.138), taillés dans deux marbres différents, permettent de supposer que la fosse était habillée de marbre. Ce placage offrait des nuances bleutées à grises sur fond blanc.

L'espace du chœur, qui formait une avancée surélevée dans la nef (bêma), était délimité par une barrière de chancel relativement simple. Parmi les douze fragments de plaques taillés dans un calcaire coquillier de grossière qualité, seulement trois sont sculptés d'une croix pattée dont on conserve à chaque fois une extrémité au contact des rubans plats délimitant les bords (fig. 23). Sur deux de ces fragments ainsi que sur le bloc LAP.MAR15.2, le ruban plat du bord est doublé par un second ruban en léger retrait. Malgré l'état fragmentaire de ces éléments, il est possible de les regrouper dans 
un ensemble cohérent grâce au type de calcaire utilisé, à une technique de taille identique - ciselure périmétrale, utilisation importante de la gradine -, la présence d'une seule face sculptée pour la plupart des éléments et des dimensions similaires. Cet ensemble est également constitué de deux fragments de pilier de chancel. Dans l'état actuel, il reste difficile de dater précisément cet ensemble qui s'inscrit dans une fourchette chronologique allant de la fin $\mathrm{du} \mathrm{IV}^{\mathrm{e}} \mathrm{s}$. au VI ${ }^{\mathrm{e}} \mathrm{s}$. On notera également la découverte d'un fragment sphérique ayant pu être une partie d'un pommeau de pilier de chancel. Taillé dans un matériau similaire, nous serions tentés de le rattacher à ce premier ensemble.

Fig. 23 - Martinšćica, fragment de plaque de chancel (cl. J. Crochat)

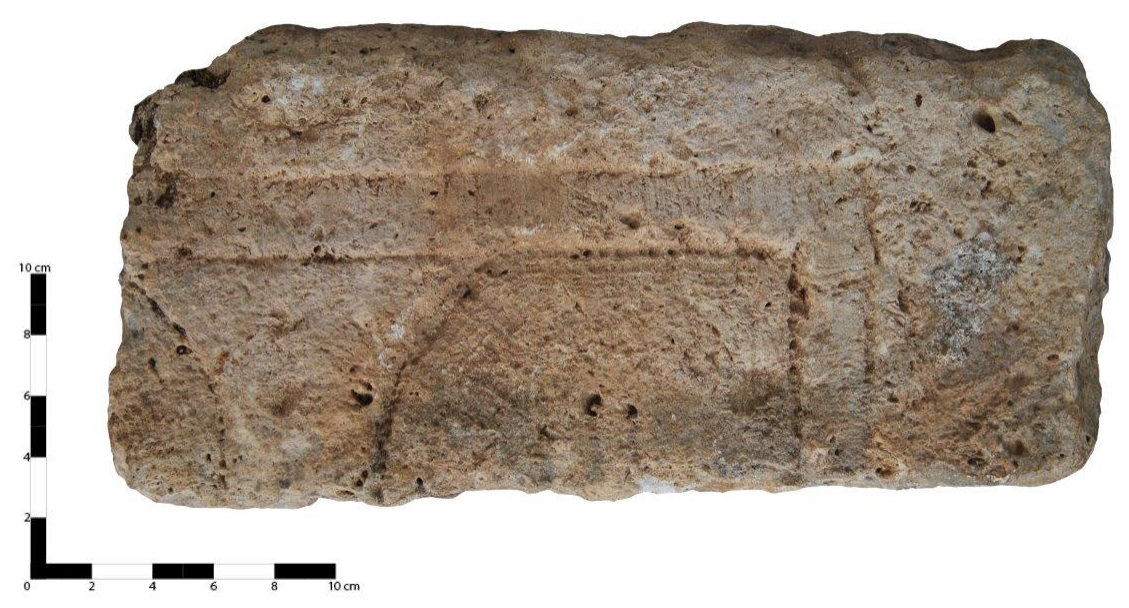

57 Un deuxième ensemble est déterminé grâce à deux fragments d'une plaque de chancel. Les motifs décorant cette plaque sont connus de Mirine, au nord de Krk, à Zadar, en passant par Osor et l'île de Rab et dateraient du V $\mathrm{V}^{\mathrm{s}}$ s. (fig. 24). La " production de l'atelier de Zadar », comme l'a nommée Pascale Chevalier ${ }^{21}$, présente sur une face une imbrication d'octogones adjacents timbrés de fleurettes. Sur la face opposée, une croix pattée domine sur un podium à quatre degrés. Pour des raisons quantitatives plusieurs plaques et piliers de chancel - nous imaginons le premier ensemble comme la barrière délimitant la bêma. Toutefois, ce n'est là qu'une hypothèse susceptible de changer au gré des découvertes à venir. 
Fig. 24 - Martinšćica, fragment de plaque de chancel (cl. et dessin J. Crochat)

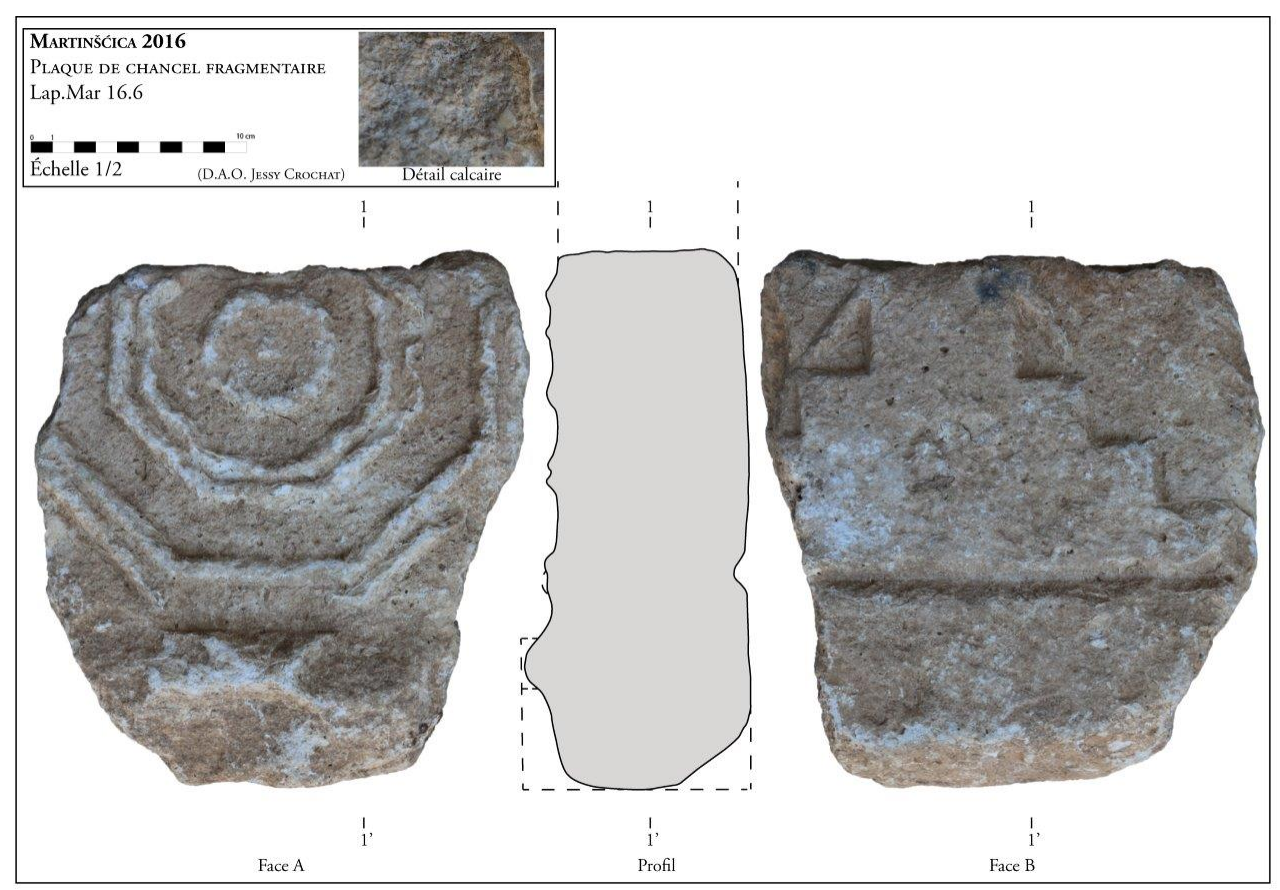

Enfin, parmi le mobilier lapidaire, ces deux années de fouilles ont mis au jour quatre fragments de colonnettes et un chapiteau. Bien qu'il soit difficile de restituer leur fonction, leurs dimensions permettent de les exclure de la sculpture architecturale. La colonnette en marbre était peut-être un pied de la table d'autel rectangulaire mais rien ne permet de l'affirmer, si bien que nous pourrions éventuellement la rattacher au chapiteau. La datation du chapiteau est complexe, faute d'exemples comparables. Le traitement des motifs, en très bas-relief, se distingue de ce que nous avons pu voir jusqu'à présent. Ce critère, ainsi que le motif à double volute entouré d'éléments végétaux, nous amène à proposer une datation relativement tardive, des $\mathrm{VII}^{\mathrm{e}}-\mathrm{VIII}^{\mathrm{e}}$ s. Les trois autres fragments, taillés dans un calcaire marbrier, appartiennent peut-être à une même colonnette. Ils pouvaient à la fois appartenir à une pergola, ou constituer les pieds d'une table d'autel. Nous manquons ici d'arguments pour pencher en faveur de l'une ou l'autre hypothèse.

\section{Des fragments de cuve}

Parmi les divers éléments découverts lors de ces deux dernières campagnes, nous retiendrons deux fragments d'une même cuve localisés dans l'espace IIIb, en lien avec un aménagement hydraulique (STR 3.111). Cette cuve présentait une ouverture circulaire dans sa partie inférieure permettant l'évacuation des liquides par la STR 3.106. Sur la paroi interne des deux fragments, un dépôt verdâtre stratifié d'environ 4 $5 \mathrm{~mm}$ d'épaisseur semble en lien avec la formation de la roche. Néanmoins, si après analyse, il ressort que nous sommes en présence d'un encroûtement tufacé, cela révélerait une activité hydraulique anthropique continue sur plusieurs années voir sur plusieurs décennies. Dans ce cas, cette cuve aurait été conçue pour recevoir un écoulement fréquent d'eau, évacué par l'ouverture inférieure. 


\section{Synthèse et perspectives d'étude}

60 Les deux premières campagnes de fouilles de l'église ont conduit à la découverte d'un déjà grand nombre de fragments lapidaire qui nous permettent d'appréhender le parti décoratif architectural de l'église, son organisation interne, mais aussi son niveau de richesse. Nous sommes en présence d'une sculpture qui montre un approvisionnement diversifié. La sculpture est une combinaison de productions à la fois locales - comme le suggère la barrière de chœur de l'église -, de productions plus régionales - à l'image de la plaque de chancel de l'atelier de Zadar - jusqu'à un approvisionnement vers des centres de distribution à l'échelle de l'empire Byzantin - comme le suggèrent les fragments de table d'autel en marbre.

61 Les prochaines campagnes de fouilles permettront, espérons-le, d'affiner les ensembles proposés. De plus, une meilleure compréhension de la chronologie du site ainsi que la comparaison avec des éléments régionaux, permettra d'affiner la datation des divers éléments mais aussi leur restitution dans l'espace.

62 Au-delà de l'enrichissement du catalogue lapidaire, une réelle réflexion doit s'engager concernant le lien entre Martinšćica et les modèles de circulation de la pierre sculptée. Celle-ci passera tout d'abord par une détermination des matériaux utilisés et, surtout, de leur provenance en lien avec une étude stylistique et iconographique qui nécessitera la confrontation de la sculpture de Martinšćica avec les sites régionaux et au-delà.

\section{Conclusion}

La seconde campagne de fouille sur la grande église du complexe de Martinšćica nous a permis de documenter des installations liturgiques élaborées - en dépit d'un médiocre état de conservation - et de recenser un mobilier liturgique de qualité, nous interpellant sur le statut de l'édifice, avec en particulier la découverte du monogramme sur un fragment de mensa en marbre ${ }^{22}$. L'analyse attentive des négatifs de mortiers, des arrachements et des vestiges de maçonneries indique que le sanctuaire était aménagé d'une plateforme de chœur (bêma), clôt d'une barrière de chancel, auquel on accédait par trois ouvertures. Un creusement en son centre correspond selon toute vraisemblance à l'emplacement d'une fosse à reliques sous l'autel, habillée d'un placage de marbre d'après des fragments de plaquette découverts. La fouille a également révélé deux sacristies sur le flanc nord de l'église. L'une d'elle conserve un magnifique pavement de mosaïques géométriques et un trou d'évacuation de l'eau dans son mur nord. La seconde, plus tardive, est accolée à l'est; elle possède un sol plus fruste de mortier, mais qui conserve le négatif d'une table dans l'angle S-E et une nouvelle évacuation d'eau dans l'angle N-E. Le sol en mortier est encore percé de petits trous de poteaux pouvant correspondre à une phase tardive. C'est assurément le cas de la première sacristie, où le sol de mosaïque est scellé par un niveau d'occupation et un foyer. Ces indices témoignent d'un très probable changement de fonction des salles annexes. Et c'est une occupation peut-être domestique que l'on tend à attribuer à l'annexe greffée contre l'abside centrale. Il est en effet désormais assuré que cette annexe, qui est pourtant établie dans une situation privilégiée avec le sanctuaire distribution directe par un passage percé dans l'abside - n'a pas de fonction funéraire. 
64 Martinšćica a fait l'objet d'un important "pillage ", qui a « décharné » l'édifice de ses matériaux décoratifs et précieux. Mais les vestiges subsistants permettent tout de même d'apprécier la qualité du mobilier et l'ampleur, comme la singularité du parti architectural.

65

À ce stade des recherches, nous travaillons sur l'hypothèse d'une villa et d'une église appartenant au domaine de l'évêque d'Osor pour les $\mathrm{V}^{\mathrm{e}}-\mathrm{VI}^{\mathrm{e}} \mathrm{s}$. À une date qui reste à déterminer et selon un premier phasage qui demande à être précisé, l'église se transforme à la fois par une rétraction de son espace liturgique et par une multiplication des annexes (fig. 25). Ce phénomène reste à apprécier, mais il pourrait traduire un changement de fonction et de statut de l'église, posant la question de l'installation d'une communauté monastique.

Fig. 25- Martinšćica, vue générale de l'église en cours de fouille (cl. M. Vuković)

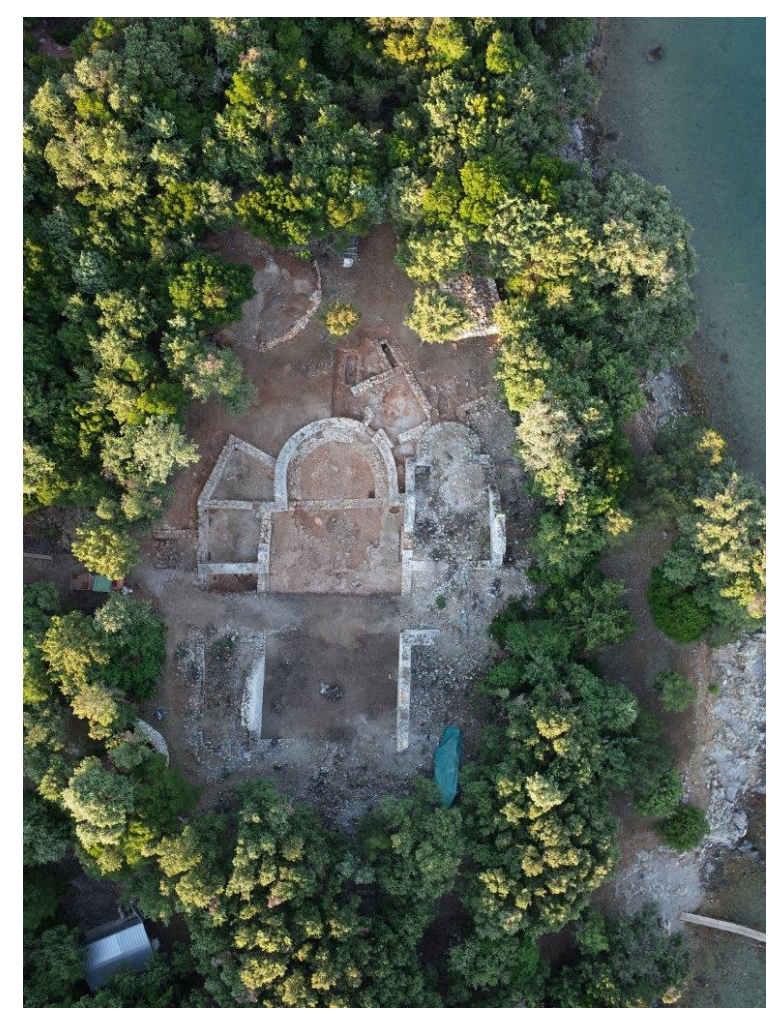

66 Ainsi, sur la base d'un postulat identique, les données archéologiques d'ores et déjà recueillies sur les sites de Mirine et de Martinšćica permettent d'ébaucher des critères de détermination du type d'occupation, et engagent de nouvelles lectures, désormais divergentes, sur leur statut et leur fonction.

67 La fouille en 2017 de la chapelle latérale sud (Martinšćica 1bis) et de l'annexe VIIIb greffée à son abside - selon une configuration et un phasage identiques à l'annexe VIIIa - devrait considérablement enrichir la documentation du site en apportant de nouvelles données sur sa fonction. Ici, et eu égard à sa situation et à sa typologie, c'est la question d'une fonction mémorielle de la chapelle qui est posée. 


\section{Premiers forages destinés aux analyses paléoenvironnementales dans les îles de Krk et de Cres (Hervé Richard, Vincent Bichet, Emilie Gauthier)}

\section{Présentation} permis de repérer certains sites susceptibles d'être explorés par des forages de reconnaissance. Une mission de terrain précédente (juin 2015) avait permis de préciser certains points et de faire un premier choix. Il s'agit de récupérer des colonnes de sédiments dans les lacs du secteur d'étude, susceptibles d'avoir conservé des signaux paléobotaniques (pollen, spores, micro-charbons, graines...) et géochimiques (indices d'érosion, variation et origine de la matière organique,...). Nous avons utilisé un carottier gravitaire Uwitec, envoyé au fond du lac depuis un bateau de type Zodiac, qui permet de remonter des carottes d'environ un mètre de longueur et d'une dizaine de centimètres de diamètre. Systématiquement, avant cette opération, une reconnaissance bathymétrique à l'aide d'un écosondeur est pratiquée afin de choisir la (ou les) zone(s) la plus favorable(s) pour effectuer le(s) forage(s).

\section{Lac d'Omišalj et baie de Sepen}

Sur l'île de Krk, le lac d'Omišalj, à côté de la ville de Njivice, a été le premier objectif de la mission. On sait que le niveau de ce lac a été artificiellement augmenté 
récemment. La reconnaissance bathymétrique a permis de reconnaitre les zones les plus profondes dans lesquelles deux carottes ont été prélevées à deux points différents $\mathrm{du}$ lac. Les premières datations radiocarbones effectuées au milieu d'une des carottes indiquent une date vers le XIV ${ }^{\mathrm{e}}$ siècle après J.-C. D'autres dates vont être faites sur la base de ces carottes afin de contrôler si la sédimentation a été suffisante pour enregistrer des niveaux contemporains des principaux sites fouillés localement.

Toujours sur l'île de Krk, le Municipium Flavium Fulfinum a été créé au fond de la baie de Sepen, au sud de l'oppidum protohistorique et de l'actuelle ville d'Omišalj. Dans les couches préparatoires du forum, l'ensemble du mobilier laisse présumer une datation vers le milieu du Ier siècle, tandis que le nom laisse supposer seulement sa finalisation et l'obtention du statut de municipe à l'époque du premier Flavien, Vespasien. Les nouvelles fouilles effectuées récemment sur le site, principalement dans la zone du forum, démontrent une continuité d'utilisation se situant désormais entre le $V^{e}$ et le VIII siècle. En l'absence de lac, d'étang ou de zone humide proches, nous avons tenté, avec le même dispositif qu'au lac d'Omišalj, de prélever les niveaux supérieurs déposés dans la baie de Sepen, occupé en partie par le port du site de Fulfinum. C'était la première fois que nous utilisions ce type de dispositif en mer. Deux carottes d'environ un mètre ont été prélevées. Une première datation ${ }^{14} \mathrm{C}$, effectuée sur des restes de bois et d'écorce triés dans les sédiments de la base d'une des carottes, indique les XIII ${ }^{\mathrm{e}}-\mathrm{XIV}{ }^{\mathrm{e}}$ siècle avant $\mathrm{J}$.-C. On peut donc supposer que ces carottes renferment des niveaux contemporains de l'occupation de Fulfinum-Mirine. D'autres datations de contrôle vont être faites prochainement ; les premiers tests paléobotaniques sont en cours.

\section{Lac d'Osor}

Sur l'île de Cres, nos recherches ont concerné les zones humides situées autour de la ville d'Osor. Cette ville connaît une occupation ininterrompue depuis l'oppidum protohistorique jusqu'à nos jours. Très proches de la ville, trois petits lacs circulaires, occupant probablement des dolines, ont été repérés. De plus, d'autres sites de même forme mais totalement comblés de sédiments organiques ont aussi été repérés dans le secteur. Deux des petits lacs, difficiles d'accès, ont fait l'objet de forages au cours de cette mission. Un seul a permis d'obtenir une carotte acceptable pour nos recherches. Le résultat des deux dates radiocarbones effectuées sur cette carotte est encourageant pour la suite. Les restes végétaux trouvés à la base donnent en effet une date située au cours du XIV ${ }^{e}$ siècle avant J.-C., ceux retrouvés au milieu de la carotte donnent une date se situant autour de l'An Mil (après J.-C.).

Les premiers tests permettant de contrôler si les sédiments recueillis contiennent suffisamment de micro-restes végétaux (pollen et spores notamment) sont en cours. Ils seront étendus aux autres carottes dans les prochaines semaines. Au préalable, une étude géophysique complète (susceptibilité magnétique, densimétrie, colorimétrie...) sera effectuée sur ces carottes. Comme indiqué, d'autres datations ${ }^{14} \mathrm{C}$ sont nécessaires pour permettre un calage chronologique précis de toutes ces carottes. Une prochaine mission devrait permettre de prélever les autres sites repérés et surtout de carotter les niveaux supérieurs du lac Vrana, situé sur l'île de Cres, dont une série d'analyses effectuées sur un carottage fait au centre du lac (publiée en 2000, Journal of Limnology) montre que le mètre supérieur de sédiment correspond aux quatre derniers 
millénaires. Une nouvelle analyse, plus précisément datée, doit aboutir à une reconstitution fine de l'évolution des rapports homme/milieux sur cette île.

L'ensemble des données recueillies déjà, ajoutés aux prochains carottages qui seront effectués dans une nouvelle mission, doit permettre d'accumuler suffisamment de données paléoenvironnementales. Une allocation de thèse (direction: H. Richard, M. Čaušević-Bully) sera demandée afin de traiter l'ensemble de ces données.

\section{NOTES}

1. Bully (S.) et Čaušević-Bully (M.), «Le monachisme insulaire dans l'archipel du Kvarner (Croatie) entre le $\mathrm{V}^{\mathrm{e}}$ et le XI ${ }^{\mathrm{e}}$ siècle. Projet de recherches archéologiques, 2011-2014 ", Bulletin du centre d'études médiévales d'Auxerre (BUCEMA), 15, Auxerre, 2011, p. 335-350 http://cem.revues.org/ index12017.html

2. Nous exprimons notre gratitude envers les financeurs de ces recherches: le ministère des Affaires Étrangères français, le ministère de la Culture croate, l'École française de Rome, la fondation Caritas Veritatis, la région de Primosko-Goranska, les communes d'Omišalj et de Mali Lošinj.

3. Čaušević-Bully (M.), Bully (S.), Archipel du Kvarner (Croatie). Prospection-inventaire des sites ecclésiaux et monastiques : campagne 2015, Chronique des activités archéologiques de l'École française de Rome, 2016. http://cefr.revues.org/1578

4. Ibid.

5. Jaksić (N.), s.v. Mirine - Omišalj, Hrvatski arheološki godišnjak, 2006, p. 297-298.

6. Documentation en cours d'élaboration.

7. Žerjal (T.), « Roman tegulae in Northern Istria », in Lipovac-Vrkljan (G.), Siljeg (B.), OzanicRoguljic (I.), Konestra (A.) éds., Rimske keramičarske i staklarske radionice. Proizvodnja i trgovina na Jadranskom prostoru. Zbornik II. međunarodnog arheološkog kolokvija Crikvenica, 28-29. listopada 2011, Crikvenica 2014, p. 227 (219-237).

8. Par A. Konestra et I. Valent.

9. Čaušević-Bully (M.), Bully (S.), «Archipel du Kvarner (Croatie). Prospection-inventaire des sites ecclésiaux et monastiques : campagne 2015 ", Chroniques des activités archéologiques de l'École française de Rome, 2016, https://cefr.revues.org/1578.

10. Les tesselles blanches sont en calcaire et les tesselles rouges en terre cuite, les "noires » paraissent taillées dans une pierre anthracite à nuances vert foncé.

11. Meder (J.), Podni mozaici u Hrvatskoj od 1. do 6. stoljeća, Zagreb, 2003, p. 34 et pl. IV-1.

12. Ibid., p. 75 et pl. XXIX-4.

13. Ibid., p. 47 et pl. XIV-3.

14. Ibid., p. 59 et pl. XXI-2.

15. Ibid., p. 69 et pl. XXVI-1.

16. Ibid., p. 117 et 120 et pl. L-1 et 5.

17. Ibid., p. 74 et pl. XXVIII-3.

18. Ibid., p. 121 et pl. LII-1.

19. Ibid., p. 31 et pl. I-4.

20. Ibid., p. 39 et pl. X-2 et 3. 
21. Chevalier (P.), Salona II. Recherches archéologiques franco-croates à Salone. Ecclesiae dalmatiae. L'architecture paléochrétienne de la province romaine de Dalmatie (IVe-VIIe S.), T. 2, Rome, École Française de Rome, 1995, p. 134.

22. En cours d'étude par P. Chevalier.

\section{INDEX}

institutions Université de Franche-Comté / UMR 6249 Chrono-Environnement (Besançon), UMR 6298 ARTeHIS (Dijon-Auxerre), École française de Rome

Mots-clés : Croatie, insularité, Antiquité tardive, haut Moyen Âge, archéologie, archéologie du bâti, monastère, complexe ecclésial, funéraire, villa

Index géographique : Croatie, Mirine, Martinšćica, Kvarner

\section{AUTEURS}

\section{MORANA ČAUŠEVIĆ-BULLY}

Université de Franche Comté, UMR 6249 Chrono-Environnement - morana.causevic[at]gmail.com SÉBASTIEN BULLY

CNRS, UMR 6298 ArTeHiS - sebastien.bully[at]club-internet.fr

\section{JESSY CROCHAT}

Archéologue, titulaire d'un Master 2 de l'université Lyon Lumière 2 - jessy.crochat[at]gmail.com

\section{PASCALE CHEVALIER}

Université de Clermont-Ferrand 2, UMR ARTEHIS- paskvalinac[at]gmail.com

\section{IVAN VALENT}

Archéologue, titulaire d'un Master 2 de l'université de Zagreb - ivan.valent[at]yahoo.com

\section{HERVÉ RICHARD}

CNRS, UMR 6249 Chrono-Environnement-herve.richard[at]univ-fcomte.fr

\section{ÉMILIE GAUTHIER}

Université de Franche Comté, UMR 6249, Chrono-Environnement-Emilie.Gauthier[at]univfcomte.fr

\section{VINCENT BICHET}

Université de Franche Comté, UMR 6249 Chrono-Environnement -vincent.bichet[at]univfcomte.fr 